\title{
A Unified Eddy-Diffusivity/Mass-Flux Approach for Modeling Atmospheric Convection
}

\author{
Kay SuselJ, Marcin J. Kurowski, AND JoAo TeiXeira \\ Jet Propulsion Laboratory, California Institute of Technology, Pasadena, California
}

(Manuscript received 15 August 2018, in final form 20 May 2019)

\begin{abstract}
A fully unified parameterization of boundary layer and moist convection (shallow and deep) is presented. The new parameterization is based on the stochastic multiplume eddy-diffusivity/mass-flux (EDMF) approach, which distinguishes between convective plumes and nonconvective mixing. The convective plumes represent both surface-forced updrafts and evaporatively driven downdrafts. The type of convection (i.e., dry, shallow, or deep) represented by the updrafts is not defined a priori, but rather depends on the near-surface updraft properties and the stochastic interactions between the plumes and the environment through lateral entrainment. Consequently, some updrafts may contribute only to the nonlocal transport within the subcloud layer, while others may condense and form shallow or even deep convection. Such a formulation is void of trigger functions and additional closures typical of modular parameterizations. The updrafts are coupled to relatively simple warm-, mixed-, and ice-phase microphysics. Each precipitating updraft forms a downdraft driven by the evaporation of detrained precipitation. The downdrafts control the development of cold pools near the surface that can invigorate convection. The new parameterization is tested in a single-column model against large-eddy simulations (LESs) for cases representing weakly precipitating marine convection and the diurnal cycle of continental deep convection. The results of these EDMF experiments compare well with the LES reference simulations. In particular, the transitions between the different dominant convection regimes are realistically simulated.
\end{abstract}

\section{Introduction}

State-of-the-art general circulation models (GCMs) cannot explicitly resolve the scales of motion smaller than $O(10-100) \mathrm{km}$, as dictated by their limited horizontal resolution. Therefore, to realistically simulate the dynamics of Earth's atmosphere, the unresolved processes have to be parameterized. In GCMs, representation of these processes is typically split into separate parameterizations for the planetary boundary layer, shallow convection, and deep convection, in addition to cloud microphysics and macrophysics (e.g., Neale et al. 2012; ECMWF 2017). Often these parameterizations are built on different theoretical foundations. For example, the bedrock of boundary layer parameterization in GCMs is the eddy-diffusivity approach (e.g., Mellor and Yamada 1974, 1982; Teixeira and Cheinet 2004; Bretherton and Park 2009). On the other hand, shallow and deep convection parameterizations heavily rely on the mass-flux approach (e.g.,

Corresponding author: Kay Suselj, kay.suselj@jpl.nasa.gov
Arakawa and Schubert 1974; Tiedtke 1989; Emanuel 1991; Bechtold et al. 2008; Wagner and Graf 2010; Yoshimura et al. 2015), which assumes that subgrid convective mixing can be represented by convective plumes embedded in a quiescent environment. Convective and large-scale cloud microphysics parameterizations are also modular. Convective microphysics is treated as part of the mass-flux model, and a separate parameterization is used for representing large-scale microphysics effects outside convective cores (e.g., Lin et al. 1983; Skamarock et al. 2008; Rienecker et al. 2008; ECMWF 2017). This general modular approach for parameterization leads to severe inconsistencies. For example, such fragmentation often calls for additional criteria for activating certain parameterizations (i.e., trigger functions), which need to be accompanied by a set of additional closure assumptions. As a result, GCMs struggle to represent gradual transitions between dominant convective regimes, for example, between shallow and deep convection, because trigger functions are often designed to switch abruptly between different parameterizations. The above problems 
contribute significantly to large uncertainties in GCM results (e.g., Jakob and Siebesma 2003). In this context, it has been suggested (e.g., Teixeira et al. 2008) that several of these coupling and modularity issues can be addressed by unifying boundary layer, shallow, and deep convection parameterizations.

To better understand the issues involved, it is instructive to study how a typical GCM represents a wellmixed subcloud layer topped by a moist convective layer. For this type of case, turbulence below the cloud base is usually parameterized with the boundary layer model, whereas moist convection aloft is described with the mass-flux parameterization. Merging these two models poses significant challenges since it is unclear how to smoothly transition from one to the other at cloud base. The problem is solved by introducing a cloud-base closure, which determines the properties of convective updrafts at cloud base and thus the boundary conditions for mass-flux parameterization. To represent the onset of shallow convection and the transition to deep convection, additional criteria for when these events occur have to be defined. Because the transition occurs gradually, the conditions for the transition are unclear (e.g., Guichard et al. 2004). As a result, GCMs have notorious difficulties correctly representing the diurnal cycle and the magnitude of continental convection (e.g., Guichard et al. 2004; Grabowski et al. 2006; Couvreux et al. 2015). Similar problems exist for other transitions associated with the evolution of the planetary boundary layer. For instance, models struggle to accurately represent the transition between the subtropical stratocumulus and shallow convection (e.g., Teixeira et al. 2011), which in turn yields significant uncertainty in the cloud radiative feedback (e.g., Bony and Dufresne 2005; Karlsson et al. 2008). Guichard et al. (2004) argued that the key to improving the representation of these transitions is a better representation of different convective regimes, including shallow and deep convection.

It appears that many, if not most, of these problems can be remedied by using a consistent subgrid-scale parameterization that represents turbulence, convection, condensation, and even microphysics, as well as their mutual interactions, within a unified framework. A number of attempts to merge the boundary layer and shallow convection parameterizations have been documented in the literature (e.g., Lock et al. 2000; Hourdin et al. 2002; Golaz et al. 2002a,b; Cheinet and Teixeira 2003; Suselj et al. 2012, 2013, 2014; Angevine et al. 2018; Suselj et al. 2019). One such approach, which has become increasingly popular, is based on the eddydiffusivity/mass-flux (EDMF) approximation (Siebesma and Teixeira 2000; Teixeira and Siebesma 2000; Soares et al. 2004; Angevine 2005; Siebesma et al. 2007; Rio and Hourdin 2008; Pergaud et al. 2009; Neggers et al. 2009; Neggers 2009; Witek et al. 2011a,b; Suselj et al. 2012, 2013; Neggers 2015; Bhattacharya et al. 2018; Tan et al. 2018; Suselj et al. 2019). Several attempts to combine shallow and deep convection within a consistent framework have also been undertaken (Bechtold et al. 2001; Kuang and Bretherton 2006; Hohenegger and Bretherton 2011; D'Andrea et al. 2014).

This paper describes a new unified parameterization that allows for a comprehensive and numerically efficient representation of atmospheric convection. It is based on an existing EDMF framework designed for simulating the boundary layer and shallow convection (Suselj et al. 2013, 2019), here extended to moist deep convection. The parameterization employs a multiplume approach in which different types of convection can coexist within the subgrid domain. An important model feature is that it facilitates continuous transitions between the dominant convective regimes, unlike more traditional approaches (e.g., Tiedtke 1989). The skill of the proposed parameterization is evaluated by means of single-column model (SCM) simulations performed for two moist convection cases, results of which are compared against large-eddy simulations (LESs). The two cases are the Rain in Cumulus over the Ocean (RICO) (van Zanten et al. 2011), representing weakly precipitating marine convection, and the LargeScale Biosphere-Atmosphere (LBA) (Grabowski et al. 2006; Kurowski et al. 2018), which represents a diurnal cycle of moist convection over land.

The paper is organized as follows. Section 2 describes the main features of the model that allow for the simulation of moist deep convection, the shallow-to-deep convection transition, and the interactions between convection and stratiform clouds. In section 3 we evaluate the new parameterization with respect to the two mentioned test cases. Finally, in section 4 we provide the summary and conclusions. The appendixes provide the additional details of the model implementation.

\section{Description of the unified EDMF model}

To represent all types of the surface-forced convection and the associated processes in a consistent manner, we develop a unified model that builds on the stochastic multiplume EDMF shallow convection model described in detail in Suselj et al. $(2013,2019)$. New components of the unified parameterization include (i) ice- and mixedphase physics, (ii) microphysical processes representing rain formation within convective updrafts, (iii) convective downdrafts driven by the evaporation of precipitation detrained from the updrafts, (iv) effects 


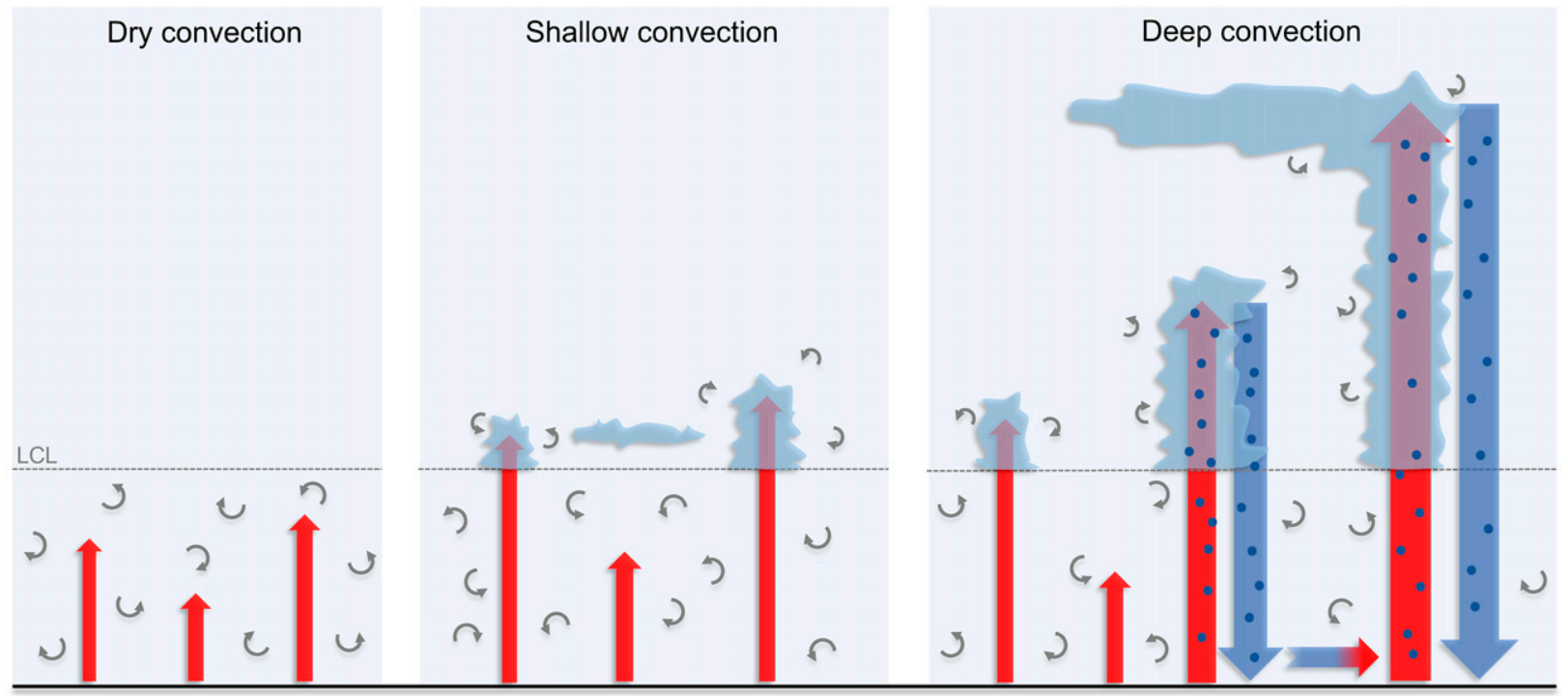

FIG. 1. Schematic of three archetypes of convection as represented by the EDMF model: (left) dry, (middle) shallow, and (right) deep convection. Vertical red arrows denote convective updrafts that can condense after reaching the lifting condensation level (LCL). Vertical blue arrows denote downdrafts associated with the precipitating updrafts. The horizontal arrow in the right panel indicates cold pools that can affect the properties of newly formed updrafts. Small gray circular arrows denote local turbulence in the nonconvective environment. Stratiform cloudiness is shown for the shallow and deep convective types.

of cold pools on convection, and (v) cloud formation in the nonconvective environment.

Figure 1 schematically illustrates three archetypal forms of atmospheric convection. These include dry convection, shallow nonprecipitating convection, and deep precipitating convection. In the tropics, they are different stages of the convective diurnal cycle over land (e.g., Guichard et al. 2004; Grabowski et al. 2006). By analogy, in the EDMF model, convection is parameterized by an ensemble of laterally entraining updrafts originating near the surface. In the dry convection stage, all the updrafts terminate before reaching their lifting condensation level. In the shallow convection stage, some of the updrafts succeed to reach their condensation level and continue rising as moist updrafts representing shallow cumulus clouds. In the model, the phase of condensed water is a function of temperature. In the deep convection stage, the updrafts can reach even higher and start to precipitate. A microphysical scheme resembling the autoconversion process represents precipitation formation. A fraction of precipitation falls through the updrafts and the rest of it detrains and forms convective downdrafts. These downdrafts are fueled by negative buoyancy production due to the evaporation of precipitation. Near the surface, the downdrafts incite the formation of cold pools. Once the cold pools intensify, they begin to enhance the growth of subsequent convective updrafts by organizing them into larger clusters, and by modifying their surface conditions. The effect of updraft clustering is represented by reducing their lateral entrainment. Positive feedback between the cold pool formation and convection promotes a rapid transition to the deep convection stage. The EDMF model also represents partial cloudiness in the nonconvective environment.

The model is designed such that (under favorable conditions) the multiple convective updrafts include dry convective updrafts and nonprecipitating and precipitating moist updrafts (that are both a natural extension of dry updrafts), all within the same subgrid domain. The termination height for each individual updraft and thus its ability to form condensation and precipitation depends only on its thermodynamic conditions and stochastic interactions with the environment.

For the remainder of this section, we provide an overview of the SCM model in which the stochastic multiplume EDMF parameterization was implemented. Next, we describe the details of the unified EDMF parameterization. The description of the numerical implementation can be found in the appendixes.

\section{a. Overview of the SCM framework}

A complete set of the SCM prognostic equations describing the state of the atmosphere includes the equations for the grid-mean moist conserved variables: total water mixing ratio $\left(q_{t}=q_{v}+q_{l}+q_{i}\right.$, where $q_{v}, q_{l}$, and $q_{i}$ are mixing ratios of water vapor, cloud liquid, and cloud ice water, respectively) and ice-liquid water 
potential temperature $\left[\theta_{l i}=\theta-\left(L_{l} q_{l}+L_{i} q_{i}\right)\left(c_{p} \pi\right)^{-1}\right.$, where $\theta$ is the potential temperature, $\pi$ the Exner function, and $L_{l}$ and $L_{i}$ the latent heats of evaporation and sublimation of water, respectively], the two horizontal components of wind speed ( $u$ and $v$ ), and turbulent kinetic energy (TKE). Water condensate is split into the cloud (i.e., suspended) and precipitating parts, of which only the cloud part is used in the definition of moist conserved thermodynamic variables. The Reynolds-averaged conservation equation for the prognostic variables in the anelastic framework can be written as

$$
\begin{aligned}
\frac{\partial \bar{\varphi}}{\partial t}= & -\underbrace{\frac{1}{\rho} \frac{\partial}{\partial z}\left(\rho \overline{w^{\prime} \varphi^{\prime}}\right)}_{\text {Subgrid vertical mixing }}-\underbrace{\sum_{\text {Other sinks or sources }}^{3} \bar{u}^{k} \frac{\partial \bar{\varphi}}{\partial x^{k}}}_{\text {Large-scale advection }}
\end{aligned}
$$

where $\varphi=\left\{\theta_{l i}, q_{t}, u, v\right\}$ represents any of the four prognostic variables, $x^{k}=\{x, y, z\}$ the two horizontal directions and one vertical direction in Cartesian coordinates, $u^{k}=\{u, v, w\}$ are the velocity components along these directions, $\rho$ is base-state dry-air density, and $S_{\varphi}$ is the source term corresponding to variable $\varphi$. In the SCM model, the source term for thermodynamic variables includes radiative cooling or heating (which is nonzero only for $\theta_{l i}$, and is here prescribed for the RICO case and set to zero for the LBA case) and microphysical source terms (which are computed in the SCM model). Following van Zanten et al. (2011), source terms for the horizontal wind speed components for the RICO case are computed from the deviation of the grid-mean wind from the geostrophic wind speed [i.e., $\bar{S}_{u}=f\left(\bar{v}-\bar{v}_{g}\right)$ and $\bar{S}_{v}=-f\left(\bar{u}-\bar{u}_{g}\right)$, where $u_{g}$ and $v_{g}$ are prescribed geostrophic wind speed components, and $f$ is the Coriolis parameter]. For the LBA case, they are prescribed as in Grabowski et al. (2006) by nudging the wind components toward their reference profile [i.e., $\bar{S}_{u}=$ $\left(\bar{u}_{\text {ref }}-\bar{u}\right) / \tau_{\text {ref }}$ and $\bar{S}_{v}=\left(\bar{v}_{\text {ref }}-\bar{v}\right) / \tau_{\text {ref }}$, where $\bar{u}_{\text {ref }}, \bar{v}_{\text {ref }}$, and $\tau_{\text {ref }}$ are prescribed reference wind speed components and nudging time scale].

A prognostic equation for TKE, which is used to model subgrid-scale motions in the nonconvective environment (see section 2d), is written as

$$
\begin{aligned}
\frac{\partial e}{\partial t}= & \underbrace{-\sum_{k=1}^{K} \bar{u}^{k} \frac{\partial e}{\partial x^{k}}}_{\text {Large-scale advection }} \underbrace{-\frac{1}{\rho} \frac{\partial}{\partial z}\left(\rho \overline{w^{\prime} e^{\prime}}\right)}_{\text {Subgrid vertical mixing }} \underbrace{-\overline{u^{\prime} w^{\prime}} \frac{\partial \bar{u}}{\partial z}-\overline{v^{\prime} w^{\prime}} \frac{\partial \bar{v}}{\partial z}}_{\text {Shear production }} \\
& \underbrace{+\frac{g}{\theta_{0}} \overline{w^{\prime} \theta_{v}^{\prime}}}_{\text {Buoyancy production }} \underbrace{-\varepsilon}_{\text {Dissipation }},
\end{aligned}
$$

where $e$ is the grid-mean TKE, and the rhs terms represent, respectively, large-scale advection, subgrid vertical mixing, shear production, buoyancy production/ consumption, and the dissipation rate. The dissipation rate $\varepsilon$ is modeled as

$$
\varepsilon=-c_{\varepsilon} \frac{e^{3 / 2}}{l_{\varepsilon}}
$$

where $c_{\varepsilon}=0.16$ is a constant, and symbol $l_{\varepsilon}$ represents the dissipation length scale, which combines two length scales as described in Suselj et al. (2019). The first length scale is the surface length scale that is proportional to the distance from the surface. The second one is defined following Mellor and Yamada (1974) and is proportional to the ratio of the first and zeroth moments of the profile of square root of TKE.

\section{b. EDMF parameterization}

The main idea behind the multiplume EDMF approach is to decompose the horizontal subgrid area into an ensemble of convective plumes and the remaining nonconvective environment. The plume ensemble consist of $N$ updrafts (denoted by $n=1, \ldots, N$ ) and $M$ downdrafts (denoted by $m=1, \ldots, M$ ). In the model, the fractional updraft area of an individual plume is small and therefore we assume a uniform distribution of the model variables within each convective plume. This is often referred as the top-hat assumption (e.g., Siebesma and Cuijpers 1995). In the nonconvective environment, the distributions of moist-conserved thermodynamic variables are assumed to be normal. These assumptions about the distributions of the model variables within the convective plumes and within the nonconvective environment are consistently used when computing the subgrid-scale fluxes and the cloud macroand microphysical processes.

In this EDMF framework, the grid-mean value of any variable $\varphi$ is the sum of area-weighted contributions from all the components at a given level:

$$
\bar{\varphi}=\sum_{n=1}^{N} a_{u_{n}} \varphi_{u_{n}}+\sum_{m=1}^{M} a_{d_{m}} \varphi_{d_{m}}+a_{e} \varphi_{e}
$$

where the subscripts $u_{n}, d_{m}$, and $e$ denote mean values from the $n$th updraft, $m$ th downdraft, and the environment, respectively, and $a_{u_{n}}, a_{d_{m}}$, and $a_{e}$ are their corresponding fractional areas. Similar equations can be written for the source terms:

$$
\bar{S}_{\varphi}=\sum_{n=1}^{N} a_{u_{n}} S_{\varphi, u_{n}}+\sum_{m=1}^{M} a_{d_{m}} S_{\varphi, d_{m}}+a_{e} S_{\varphi, e}
$$


where $S_{\varphi, u_{n}}, S_{\varphi, d_{m}}$, and $S_{\varphi, e}$ represent the source terms for $\varphi$ in the $n$th updraft, $m$ th downdraft, and environment, respectively. By definition, the sum of all fractional areas equals unity:

$$
\sum_{n=1}^{N} a_{u_{n}}+\sum_{m=1}^{M} a_{d_{m}}+a_{e}=1 .
$$

Following Suselj et al. (2019) the subgrid-scale fluxes are computed as a sum of four components:

$$
\begin{aligned}
\overline{w^{\prime} \varphi^{\prime}}= & \underbrace{\left.a_{e} \overline{w^{\prime} \varphi^{\prime}}\right|_{e}}_{\text {Eddy diffusivity }}+\underbrace{\sum_{n=1}^{N} a_{u_{n}}\left(\varphi_{u_{n}}-\bar{\varphi}\right)\left(w_{u_{n}}-\bar{w}\right)}_{\text {Updraft MF }} \\
& +\underbrace{\sum_{m=1}^{M} a_{d_{m}}\left(\varphi_{d_{m}}-\bar{\varphi}\right)\left(w_{d_{m}}-\bar{w}\right)}_{\text {Downdraft MF }} \\
& +\underbrace{a_{e}\left(\varphi_{e}-\bar{\varphi}\right)\left(w_{e}-\bar{w}\right)}_{\text {Environmental MF }},
\end{aligned}
$$

where the rhs terms represent, respectively, local turbulence in the environment, mass flux from the convective updrafts, mass flux from the convective downdrafts, and mass flux from the subsiding environment. Because the local turbulence in the environment is modeled with the downgradient eddy-diffusivity approach (see below) it is usually referred to as the eddydiffusivity term. Given the assumption that the plumes are horizontally homogeneous, Eq. (7) represents the exact decomposition of the subgrid fluxes into the four components (e.g., Suselj et al. 2019).

Local turbulence in the environment is parameterized as

$$
\left.\overline{w^{\prime} \varphi^{\prime}}\right|_{e}=-l_{\varphi} \sqrt{e} \frac{\partial \bar{\varphi}}{\partial z}
$$

where $l_{\varphi}$ is the mixing length that combines the surfacelayer mixing length and the TKE-based mixing length (Teixeira and Cheinet 2004), weighted by a function of Richardson number. The model distinguishes between the mixing lengths for the momentum components and for the thermodynamic variables so that their ratio (which can be interpreted as the Prandtl number) follows the stability dependence from the quasi-normal scale elimination theory (Sukoriansky et al. 2005, 2006). A detailed formulation of $l_{\varphi}$ can be found in Suselj et al. (2019). Equation (8) is a simplification of the one from Suselj et al. (2019). Here the subgrid flux of variable $\varphi$ is proportional to the vertical gradient of its grid-mean value, while in Suselj et al. (2019) it is proportional to the vertical gradient of $\varphi$ in the nonconvective environment.
These two formulations yield similar fluxes when the cumulative fractional area of convective plumes is small.

For simplicity, the subgrid transport of TKE is modeled with the eddy-diffusivity approximation:

$$
\overline{w^{\prime} e^{\prime}}=-l_{e} \sqrt{e} \frac{\partial e}{\partial z}
$$

where $l_{e}$ equals the mixing length for momentum. For testing purposes, we also modified the TKE transport to include the mass-flux terms, but we did not see any significant change. Therefore, the additional mass-flux terms are omitted in the main formulation.

\section{c. Representation of liquid, mixed, and ice phases}

The phase of water condensate (i.e., liquid, mixed, or ice) is assumed to be only a function of temperature (Grabowski 1998). The liquid phase is exclusively present above the freezing temperature $\left(T_{\max }=273 \mathrm{~K}\right)$, the ice phase is exclusively present below $T_{\min }=233 \mathrm{~K}$, and the mixed phase exists in between these two limits. For the mixed phase, the fraction of liquid water is a linear function of temperature. For example, the fraction of cloud liquid water $r_{l}$ in the mixture is given by

$$
r_{l} \equiv \frac{q_{l}}{q_{l}+q_{i}}= \begin{cases}0, & T<T_{\min } \\ \frac{T-T_{\min }}{T_{\max }-T_{\min }}, & T_{\min } \leq T \leq T_{\max } \\ 1, & T>T_{\max } .\end{cases}
$$

The latent heat and the saturated mixing ratio of the mixture are both taken as the weighted averages of the ice and liquid parts: $q_{s}=q_{s l} r_{l}+\left(1-r_{l}\right) q_{s i}$ and $L=L_{l} r_{l}+\left(1-r_{l}\right) L_{i}$, where $q_{s l}$ and $L_{l}$ are the saturated water mixing ratio and the latent heat for liquid water, and $q_{s i}$ and $L_{i}$ for ice, respectively. We use the same function to determine the phase of precipitating water. A practical consequence of this simple approach is that only one variable is needed to describe the two phases. Similar temperature-dependent formulations are used in many climate models (e.g., Cesana et al. 2015) in lieu of parameterizing detailed processes responsible for freezing and melting of rain and cloud water.

\section{d. Convective updrafts}

Convective updrafts are represented by an ensemble of laterally entraining steady-state plumes initiated at the surface. The thermodynamic and dynamic properties of the $n$th updraft $\varphi_{u}=\left\{\theta_{l i, u_{n}}, q_{t, u_{n}}, u_{u_{n}}, v_{u_{n}}\right\}$ are described by the following differential equation (Suselj et al. 2013, 2019): 


$$
\frac{\partial \varphi_{u_{n}}}{\partial z}=\varepsilon_{u_{n}}\left(\bar{\varphi}-\varphi_{u_{n}}\right)+\frac{S_{\varphi, u_{n}}}{w_{u_{n}}},
$$

where $\varepsilon_{u_{n}}$ is the entrainment rate, $S_{\varphi, u_{n}}$ the sum of source terms for $\varphi$ [which in the current model are due to autoconversion of cloud water into rainwater, evaporation of precipitating water, and latent heat release (consumption) associated with melting (freezing) of precipitating ice (liquid) water], and $w_{u_{n}}$ is the vertical velocity of the $n$th updraft. Equation (11) assumes that updrafts entrain the environmental air with the properties of the grid-mean fields, which is consistent with Kurowski et al. (2019). In the current model, the number of updrafts is fixed to 20 per time step $(N=20)$, which we find to be sufficient for simulating the diversity among convective updrafts. In section 3 we study the sensitivity to the number of updrafts in the current SCM implementation. We expect that the required number of updrafts may depend on the temporal resolution of the model, with a larger ensemble required for longer time steps. For each of the updrafts, a set of equations describing its properties is independently integrated from the surface upward as long as the updraft vertical velocity $w_{u_{n}}$ remains positive. At the level where the updraft vertical velocity becomes zero the updraft is assumed to terminate.

The steady-state equation for the updraft velocity is similar to Eq. (11) (e.g., de Roode et al. 2012):

$$
\frac{1}{2} \frac{\partial w_{u_{n}}^{2}}{\partial z}=a_{w} B_{u_{n}}-b_{w} \varepsilon_{u_{n}} w_{u_{n}}^{2},
$$

where $a_{w}=1$ and $b_{w}=1.5$ are model constants accounting for turbulent drag and pressure perturbation as in Suselj et al. (2013, 2019), and are one of the possible combination suggested by de Roode et al. (2012). The updraft buoyancy is $B_{u_{n}}=g\left(\theta_{v, u_{n}} / \bar{\theta}_{v}-1\right)$, where $\theta_{v}=$ $\theta\left[1+0.61 q_{v}-\left(q_{l}+q_{i}\right)\right]$ is the virtual potential temperature. Since we assume a uniform distribution of the variables within the updrafts, an updraft is fully cloudy if the total water $q_{t, u_{n}}$ exceeds the saturation threshold $q_{s, u_{n}}$, or clear otherwise. The excess of water vapor over the saturation threshold is converted into cloud water $\left[q_{c, u_{n}}=\max \left(q_{t, u_{n}}-q_{s, u_{n}}, 0\right)\right.$, where $q_{c}$ is the sum of the liquid and ice water mixing ratios].

The equations for the horizontal wind components in the updrafts are the same as in Suselj et al. (2019). The source term due to the wind drag is parameterized as $S_{u, u_{n}} / w_{u_{n}}=-2 / 3 \varepsilon_{u_{n}}\left(\bar{u}-u_{u_{n}}\right)$ following Romps (2012). The final form of the equation for the $u$ component is

$$
\frac{\partial u_{u_{n}}}{\partial z}=\frac{\varepsilon_{u_{n}}}{3}\left(\bar{u}-u_{u_{n}}\right)
$$

and the same form is used for the $v$ component. Effectively, the entrainment rate is reduced 3 times compared to scalars.

To integrate the updraft properties in the vertical [Eqs. (11)-(13)] one needs to determine the lower boundary conditions. We assume that convection is triggered in the surface layer where the updrafts are formed by the most energetic air parcels (i.e., those with the highest vertical velocity). Measurements suggest that the near-surface vertical velocity, total water mixing ratio, and virtual potential temperature are all positively correlated in the surface layer (e.g., Mahrt and Paumier 1984). Therefore, we prescribe a jointnormal distribution of these variables near the surface. The boundary conditions for the updraft properties are taken from the right tail of the near-surface velocity distribution. In this model, the updrafts are assumed to represent the near-surface vertical velocity between one and three standard deviations, which results in a fixed total surface updraft area of $15.7 \%$. In our previous work (Suselj et al. 2019), we showed that the EDMF model has little sensitivity to the value of surface updraft area, as long as the surface updraft area is larger than approximately $5 \%$. In the subcloud layer, this is because of the compensation of subgrid fluxes represented by the four components, and above the cloud base because at the cloud base the fractional area of updrafts that condense and penetrate into the cloud layer stays fairly constant regardless of surface updraft area. The surface vertical velocity for each updraft is computed by discretizing the tail of the normal distribution into $N$ equidistant bins, and associating the mean value of each bin with the corresponding updraft. The total water mixing ratio and virtual potential temperature for each of the updrafts are computed by integrating the joint-normal distribution over the velocity bins. The joint probability density function (PDF) is characterized as follows. The first moments of the distributions match the grid-mean values of the surface thermodynamic variables. The second moments are parameterized as the functions of the surface-layer turbulent scales and are modified when the cold pool effects become significant (see section $2 \mathrm{~g}$ for details). Except for this last modification, the approach essentially follows Cheinet (2003, 2004).

The stochastic entrainment rate is parameterized assuming that the net effect of lateral entrainment can be represented as a superposition of discrete entrainment events of constant rate (Romps and Kuang 2010). For the updraft that rises a distance $d z$, a probability of an entrainment event is represented by the binomial distribution and is proportional to $d z / L_{\varepsilon}$, where $L_{\varepsilon}$ is the distance at which the rising updraft entrains once on 
average. When the updraft travels a finite height $\Delta z$, the number of entrainment events is drawn from the Poisson distribution, as it represents the superposition of independent binomial distributions. The average entrainment rate $\varepsilon$ over $\Delta z$ (which in the model is taken as the depth between two model layers) for the $n$th updraft is computed as

$$
\varepsilon_{u_{n}}(\Delta z)=\frac{\varepsilon_{0}}{\Delta z} \mathscr{P}\left(\frac{\Delta z}{L_{\varepsilon}}\right),
$$

where $\varepsilon_{0}=0.2$ represents fractional mass of the entrained air in a single entrainment event, the parameterization of height $L_{\varepsilon}$ is described in section $2 \mathrm{~g}$, and $\mathscr{P}(\lambda)$ represents a random number drawn from the Poisson distribution with the parameter $\lambda$. Each updraft is associated with its own profile of entrainment rates. This is because for each of the updrafts (and for each vertical level of the model) the entrainment rate is calculated as an independent realization of Eq. (14).

Individual updrafts are characterized by unique surface conditions and entrainment rate profiles, therefore their thermodynamic properties and termination heights differ. Termination height is an important updraft property, as it controls whether the updraft represents only dry convection, or it can develop to moist nonprecipitating or even precipitating convection. In Suselj et al. (2019) we investigated which of the two factors controlling their development (i.e., surface conditions or lateral entrainment) is more responsible for terminating the updrafts at different heights for shallow convection. Here, we extend that analysis by investigating the role of these two factors for precipitating convection (section 3 ).

\section{e. Microphysical processes in updrafts}

In addition to condensation and evaporation, three microphysical processes are considered for the updrafts: conversion of cloud water into precipitating water, evaporation of precipitating water in the dry part of an updraft, and the latent heat release due to melting/ freezing of the ice/liquid precipitation. These processes contribute to the source terms of moist conserved thermodynamic variables since precipitating water is excluded from their definition.

The formulation of the conversion rate of cloud water into precipitating water closely follows D'Andrea et al. (2014), and generally resembles the autoconversion parameterization of Kessler (1969). It assumes that the excess of cloud water above a threshold $q_{0}$ is converted into rainwater with a prescribed time scale $\tau_{p, u_{n}}$. Therefore, the source term due to autoconversion for the total water mixing ratio within an updraft is

$$
S_{q_{t}, u_{n}}^{\mathrm{ac}}=-\max \left(\frac{q_{c, u_{n}}-q_{0}}{\tau_{p, u_{n}}}, 0\right),
$$

where $q_{0}=1.25 \times 10^{-3} \mathrm{~kg} \mathrm{~kg}^{-1}$ is close to the values used in other works (e.g., D'Andrea et al. 2014; Park and Bretherton 2009; Hohenegger and Bretherton 2011). Park and Bretherton (2009) used a similar parameterization for shallow convection, except that in their model the time scale $\tau_{p, u_{n}}$ is assumed to be small and therefore the excess of cloud water is instantly converted into rain. Hohenegger and Bretherton (2011) used the parameterization of Park and Bretherton (2009) for simulating deep convection.

For each updraft, the autoconversion time scale $\tau_{p, u_{n}}$ depends on its vertical extent $\Delta p_{\mathrm{cld}, u_{n}}$ :

$$
\tau_{p, u_{n}}= \begin{cases}\infty, & \Delta p_{\mathrm{cld}, u_{n}}<p_{\min } \\ \tau_{p 0}\left(\frac{\Delta p_{\mathrm{cld}, u_{n}}-p_{\min }}{p_{\max }-p_{\min }}\right)^{-1}, & p_{\min } \leq \Delta p_{\mathrm{cld}, u_{n}} \leq p_{\max }, \\ \tau_{p 0}, & \Delta p_{\mathrm{cld}, u_{n}}>p_{\max },\end{cases}
$$

where $\tau_{p 0}=15 \mathrm{~s}$, and $p_{\min }=150 \mathrm{hPa}$ represents a minimal cloud thickness for which precipitation initiates. The time scale decreases with increasing cloud thickness until it reaches $\tau_{p 0}$ at $p_{\max }=500 \mathrm{hPa}$. The fixed minimum and maximum thicknesses for precipitation initiation are imposed instead of representing more explicitly complex physical processes that lead to precipitation formation. The proposed minimum thickness agrees well with observations (e.g., Rangno and Hobbs 2005) and similar values are also used in other parameterizations (e.g., Gregory and Rowntree 1990; Tiedtke 1989; D'Andrea et al. 2014).

We assume that a fraction of precipitating water falls within the updraft and the rest of it is detrained into the associated downdraft. Below the cloud base, where the updraft is unsaturated, precipitating water is subject to evaporation as in Boville et al. (2006). The source term for the total water mixing ratio due to precipitation evaporation is defined as

$$
S_{q_{t}, u_{n}}^{\mathrm{ev}}=\max \left[k_{e}\left(1-\frac{q_{v, u_{n}}}{q_{s, u_{n}}}\right) \sqrt{\mathrm{RR}_{u_{n}}}, 0\right],
$$

where $k_{e}=2.5 \times 10^{-4}, q_{v, u_{n}}$ and $q_{s, u_{n}}$ are the water vapor and saturated water vapor mixing ratios in the $n$th updraft, and $\mathrm{RR}_{u_{n}}$ is the precipitation rate (i.e., the flux of precipitating water in $\mathrm{kg} \mathrm{m}^{-2} \mathrm{~s}^{-1}$ ) in the $n$th updraft. 
The precipitation rate $\mathrm{RR}_{u_{n}}$ in the $n$th updraft is computed assuming a balance between the vertical divergence of the precipitation flux, its sources, and sinks (e.g., Morrison and Gettelman 2008):

$$
\frac{\partial \mathrm{RR}_{u_{n}}}{\partial z}=\rho\left[S_{q_{t}, u_{n}}^{\mathrm{ac}}\left(1-f_{p}\right)+S_{q_{t}, u_{n}}^{\mathrm{ev}}\right],
$$

where $f_{p}$ denotes a fraction of rain produced at a certain height that is detrained into the complementary downdraft, and $S_{q_{t}, u_{n}}^{\mathrm{ac}}$ and $S_{q_{t}, u_{n}}^{\mathrm{ev}}$ are the source and sink terms for precipitation formation and evaporation [Eqs. (15) and (17)], respectively. For simplicity, we set the parameter $f_{p}$ to a constant value of 0.5 . Future work is needed to improve this element of the parameterization.

The phase of precipitating water is always assumed to be a function of temperature, so that the fraction of liquid precipitation follows $r_{l}$ from Eq. (10). Latent heat release due to melting/freezing of the precipitating water can thus be written as

$$
S_{\theta_{l i}, u_{n}}^{\text {melt }}=-\frac{L_{l}-L_{i}}{\rho c_{p} \pi} \operatorname{RR}_{u_{n}} \frac{\partial r_{l}}{\partial z},
$$

where $\mathrm{RR}_{u_{n}} \partial r_{l} / \partial z$ represents the rate of melting/freezing of precipitation at a certain height, $L_{l}-L_{i}$ the latent heat of freezing, and the product of $\rho c_{p} \pi$ the effect of the latent heat release on sensible heat.

In the final form, the source terms for the ice-liquid water potential temperature in the updrafts include the three processes:

$$
S_{\theta_{l i}, u_{n}}=-\frac{L}{c_{p} \pi}\left(S_{q_{t}, u_{n}}^{\mathrm{ac}}+S_{q_{t}, u_{n}}^{\mathrm{ev}}\right)+S_{\theta_{l i}, u_{n}}^{\mathrm{melt}},
$$

while for the total water mixing ratio only two of them contribute:

$$
S_{q_{t}, u_{n}}=S_{q_{t}, u_{n}}^{\mathrm{ac}}+S_{q_{t}, u_{n}}^{\mathrm{ev}}
$$

To efficiently solve the updraft equations, the source terms representing the evaporation and/or melting of precipitation as well as the precipitation water loading effect on updraft buoyancy are neglected when computing the updraft thermodynamic and dynamic properties (see appendix A for details).

\section{f. Convective downdrafts}

For each of the precipitating updrafts, we create a complementary downdraft that is devoid of cloud water but contains some precipitating water detrained from the associated updraft. Evaporative cooling of that precipitation is the key source of (negative) buoyancy driving the downdraft. The equations governing the thermodynamic and dynamic properties of the $m$ th downdraft $\varphi_{d_{m}}$ have a form:

$$
\frac{\partial \varphi_{d_{m}}}{\partial z}=-\varepsilon_{d_{m}}\left(\bar{\varphi}-\varphi_{d_{m}}\right)-\frac{S_{\varphi, d_{m}}}{w_{d_{m}}}
$$

for $\varphi_{d_{m}}=\left\{\theta_{l i, d_{m}}, q_{t, d_{m}}, u_{d_{m}}, v_{d_{m}}\right\}$. The downdraft equations are essentially the same as the updraft equations [Eq. (11)] but with the opposite sign for the rhs terms owing to the fact that downdraft velocity is negative. The $m$ th downdraft is initialized at one model level below the highest level at which precipitation in the complementary $n$th updraft is formed [i.e., one level below the highest level where $S_{q_{t}, u_{n}}^{\text {ac }}$ in Eq. (15) is greater than zero]. At the initialization level, the vertical velocity is the same as for the complementary updraft but with a negative sign. For simplicity, the boundary conditions for the thermodynamic variables and the horizontal wind components follow the grid-mean model values. The fractional area and the profile of the entrainment rate are both assumed to be the same as for the complementary updraft. Similarly to the updrafts, the source terms for the horizontal velocity components reduce an effective entrainment rate. The only two source terms for the thermodynamic variables in the downdrafts are the evaporation of precipitation and melting/freezing of precipitating water, whose formulations are essentially the same as for the updrafts [Eqs. (17) and (19), respectively]:

$$
\begin{aligned}
& S_{q_{t}, d_{m}}^{\mathrm{ev}}=\max \left[k_{e}\left(1-\frac{q_{v, d_{m}}}{q_{s, d_{m}}}\right) \sqrt{\mathrm{RR}_{d_{m}}}, 0\right], \\
& S_{\theta_{l i}, d_{m}}^{\mathrm{ev}}=-\frac{L}{c_{p} \pi} S_{q_{t}, d_{m}},
\end{aligned}
$$

and

$$
S_{\theta_{l i}, d_{m}}^{\text {melt }}=-\frac{L_{l}-L_{i}}{\rho c_{p} \pi} \operatorname{RR}_{d_{m}} \frac{\partial r_{l}}{\partial z} .
$$

The precipitation rate in the $m$ th downdraft is calculated as for the updrafts [Eq. (18)] assuming the balance between the precipitating source terms and the divergence of the precipitation rate:

$$
\frac{\partial \mathrm{RR}_{d_{m}}}{\partial z}=\rho\left(S_{q_{t}, u_{n}}^{\mathrm{ac}} f_{p}+S_{q_{t}, d_{m}}^{\mathrm{ev}}\right),
$$

where the first term on the rhs represents the rate of precipitation detrained from the $n$th updraft into the $m$ th downdraft, and the second term represents the evaporation of precipitation within the downdraft. 
The equation for the vertical velocity in the $m$ th downdraft is given by

$$
\frac{1}{2} \frac{\partial w_{d_{m}}^{2}}{\partial z}=a_{w} B_{d_{m}}+\left(b_{w} \varepsilon_{d_{m}}+p_{w}\right) w_{d_{m}}^{2},
$$

where $p_{w}$ encapsulates the effects of the dynamic pressure below the downdraft as it approaches the surface. Adding this term assures that the downdrafts gradually slow down as they approach the surface and prevents large vertical velocity gradients between the surface and the first model level. The excessively large gradients of the vertical velocity would imply unreasonably large tendencies of the prognostic variables from the massflux contributions within the downdrafts. Similarly to Gerard (2007), $p_{w}$ is parameterized as

$$
p_{w}= \begin{cases}\frac{1-\exp \left(z / z_{00}-1\right)}{2 z}, & z \leq z_{00}, \\ 0, & z>z_{00}\end{cases}
$$

where $z_{00}=10^{3} \mathrm{~m}$ represents the height at which the downdraft starts to feel the effects of surface proximity. In the model, the downdrafts terminate only at the surface. The downdraft vertical velocity is assumed to be always negative, with a minimum magnitude of $-10^{-2} \mathrm{~m} \mathrm{~s}^{-1}$ assuring that all downdrafts reach the surface. For downdrafts terminating somewhere above the surface an additional parameterization for the sedimentation and evaporation of precipitation below the termination level would be required, increasing the complexity of the scheme. We confirmed that the results are not sensitive to the exact value of the threshold.

\section{g. Parameterization of cold pool effects}

An important process associated with precipitating convection is the development of cold pools and their impact on the deepening of convective clouds. Cold pools are formed by penetrative downdrafts transporting cooler and drier air from the middle troposphere into the boundary layer. They are responsible for the formation of moist buoyant patches associated with the wind convergence, over which subsequent convection can develop (e.g., Khairoutdinov and Randall 2006; Jeevanjee and Romps 2013; Schlemmer and Hohenegger 2014). Furthermore, cold pools aggregate convective plumes that become less exposed to the environment and reduce their lateral entrainment (e.g., Kuang and Bretherton 2006; Khairoutdinov and Randall 2006). These are considered the most significant effects that are included in our parameterization. We represent them by introducing an additional dependency of the entrainment rate and the near-surface conditions on the strength of cold pools. We first define a new parameter, a vertical velocity scale $w_{D}$, meant to represent the strength of cold pools:

$$
w_{D}(t)=\frac{\alpha_{D}}{\tau_{D}} \int_{t-\tau_{D}}^{t}\left(\frac{1}{z_{\mathrm{cb}}} \int_{0}^{z_{\mathrm{cb}}} \sum_{n=1}^{N}\left|w_{d_{n}} a_{d_{n}}\right| d z\right) d t,
$$

where the time scale $\tau_{D}=4 \mathrm{~h}, z_{\mathrm{cb}}$ is the cloud-base height, $\alpha_{D}=1.4 \times 10^{3}$ is a parameter, and $\sum_{n=1}^{N}\left|w_{d_{n}} a_{d_{n}}\right|$ is the magnitude of the mass flux from all $N$ downdrafts. In this formulation, $w_{D}$ is a function of the net mass flux in the subcloud layer averaged over the preceding time $\tau_{D}$. The current formulation encapsulates the following key findings from Schlemmer and Hohenegger (2014). First, the development of cold pools is associated with convective downdrafts near the surface. Second, the growth and strengthening of cold pools continue for a few hours after the peak of convection. This is considered in Eq. (29) by temporal integration over $\tau_{D}$, which has a similar meaning and value to those of the relaxation scale for the convection organization parameter from Mapes and Neale (2011).

In the model, the velocity scale $w_{D}$ modifies the lateral entrainment by modulating the entrainment length scale $L_{\varepsilon}$ [Eq. (14)] when its value exceeds the surface velocity scale. The entrainment length scale is formulated as

$$
L_{\varepsilon}=\phi \sqrt{z_{\mathrm{top}}} \max \left(\frac{w_{D}}{w_{*}}, 1\right),
$$

where $\phi=2.5 \mathrm{~m}^{1 / 2}$ is a constant, $z_{\text {top }}$ is the depth of the moist convective layer (taken as the depth of the single test plume initialized at the surface and with near-zero entrainment rate) and $w_{*}$ is the Deardorff surface velocity scale. Equation (14) shows that larger values of $L_{\varepsilon}$ are on average associated with smaller entrainment rates. Consistent with previous studies (e.g., Böing et al. 2012), this parameterization assumes that deeper convective clouds tend to be wider and therefore should on average entrain less. Schlemmer and Hohenegger (2014) showed that for a growing phase of convection the strength of cold pools is associated with wider convective updrafts featuring reduced entrainment. Both of these observations are encapsulated in Eq. (30).

The other parameterized effect of cold pools is a modification of the near-surface atmospheric conditions, which control the boundary conditions for the updrafts. We modify the standard deviations of the nearsurface PDFs for the vertical velocity and thermodynamic variables to simulate their increased values due to 
the cold pool activity (e.g., Kurowski et al. 2018). In essence, they become functions of $w_{D}$ :

$$
\sigma_{\varphi}=\sigma_{\varphi, *} \max \left(\frac{w_{D}}{w_{*}}, 1\right),
$$

where $\varphi=\left\{w, q_{t}, \theta_{v}\right\}$ and $\sigma_{\varphi, *}$ are the standard deviations derived from the surface-layer scaling laws (Suselj et al. 2019):

$$
\begin{aligned}
\sigma_{w, *} & =\alpha_{w} w_{*}, \\
\sigma_{q_{t}, *} & =\alpha_{q_{t}} \frac{\left.\overline{w^{\prime} q_{t}^{\prime}}\right|_{s}}{w_{*}}, \\
\sigma_{\theta_{v}, *} & =\alpha_{\theta_{v}} \frac{\left.\overline{w^{\prime} \theta_{v}^{\prime}}\right|_{s}}{w_{*}},
\end{aligned}
$$

where $\alpha_{w}=0.57$ and $\alpha_{q_{t}}=\alpha_{\theta_{v}}=2.9$. Since the surface updraft conditions are associated with the tails of these distributions, the increased variances help to produce more buoyant plumes with larger surface-layer vertical velocities.

\section{h. Condensation and cloud formation in the nonconvective environment}

The new parameterization represents cloud formation in the environment using the exact same formulation as for the updrafts, but for the distribution-integrated quantities. The current version does not yet include the microphysical processes describing precipitation formation within stratiform clouds. As a first step, we compute the mean values of the moist conserved variables in the environment combining Eqs. (4) and (6):

$$
\begin{aligned}
\varphi_{e}= & \left(\bar{\varphi}-\sum_{m=1}^{M} \varphi_{u_{m}} a_{u_{m}}-\sum_{n=1}^{N} \varphi_{d_{n}} a_{d_{n}}\right) \\
& \times\left(1-\sum_{m=1}^{M} a_{u_{m}}-\sum_{n=1}^{N} a_{d_{n}}\right)^{-1} .
\end{aligned}
$$

Next, following the assumption of normal distributions of the environmental variables, we use a cloud scheme based on the PDF (e.g., Mellor 1977; Sommeria and Deardorff 1977; Bechtold et al. 1995; Cheinet and Teixeira 2003) to calculate both cloud fraction and the associated condensed water mixing ratio. For the cloud fraction, one needs to integrate the joint-normal PDF of $\theta_{l i}$ and $q_{t}$ over the region where the total water mixing ratio exceeds its saturation value. The condensed water mixing ratio is obtained through a similar integral, except that it is weighted by the difference between the total water mixing ratio and the saturated water vapor mixing ratio. The final expressions for the cloud fraction and the cloud water mixing ratio in the environment are

$$
\mathrm{CC}_{e}=0.5\left[1+\operatorname{erf}\left(\frac{s_{e}}{\sqrt{2} \sigma_{s, e}}\right)\right]
$$

and

$$
q_{c, e}=2 \sigma_{s, e}\left[\mathrm{CC}_{e} \frac{s_{e}}{\sigma_{s, e}}+\frac{1}{\sqrt{2 \pi}} e^{s_{e}^{2} /\left(2 \sigma_{s, e}^{2}\right)}\right],
$$

where $s_{e}=q_{t, e}-q_{s, e}\left(\theta_{l i, e}, p\right)$ is the mean saturation deficit or excess and $\sigma_{s, e}$ is its standard deviation. The latter is computed from the second moments of the moist conserved thermodynamic variables:

$$
\sigma_{s, e}=\frac{a}{2}\left(\left.\overline{q_{t}^{\prime} q_{t}^{\prime}}\right|_{e}-2 \alpha \overline{\left.q_{t}^{\prime} \theta_{l i}^{\prime}\right|_{e}}+\left.\alpha^{2} \overline{\theta_{l i}^{\prime} \theta_{l i}^{\prime}}\right|_{e}\right)^{1 / 2},
$$

with $\alpha$ and $a$ defined as in Cheinet and Teixeira (2003). The second moments are parameterized as in Bechtold et al. (1995), assuming local balance between their turbulent production and dissipation. Although this parameterization was originally developed for warm boundary layer clouds, we also use it to diagnose mixed-phase and ice clouds. The current version of the cloud scheme does not allow for supersaturation over ice, which may play an important role in the cloud development. In particular, the development of high-level cirrus clouds is known to be delayed until a significant supersaturation is reached (e.g., Lohmann and Karcher 2002). Parameterization of the second moments will be improved in future work, as Khairoutdinov and Randall (2002) showed that for precipitating convection the second moment budgets are shaped by the covariances between thermodynamic variables and rain rates.

\section{Results}

The EDMF parameterization is tested in the SCM framework for the cases representing (i) weakly precipitating marine convection (RICO case) and (ii) the diurnal cycle of continental precipitating convection (LBA case). To investigate the impact of the stochastic entrainment parameterization on convective plumes, we create an ensemble of 10 EDMF realizations for each case and analyze their mean and interquartile range values. To understand how the key processes (such as precipitation formation and evaporation and cold pools) shape the thermodynamic structure of the atmosphere, we perform multiple simulations in which we modify the parameterization of these processes. 

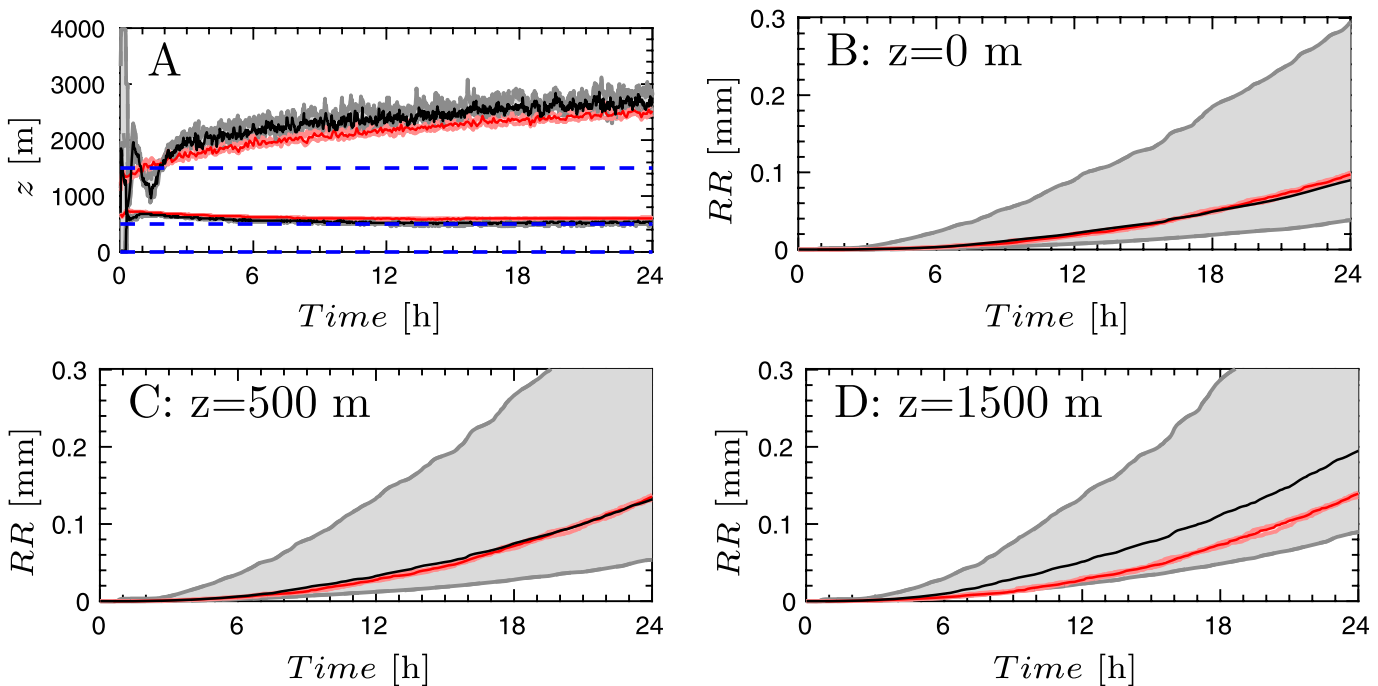

EDMF

LES

FIG. 2. RICO case. Time series of (a) cloud-base and cloud-top heights, and cumulative precipitation (b) at the surface, (c) just below cloud base (at $500 \mathrm{~m}$ ), and (d) in the central part of cloud layer (at $1500 \mathrm{~m}$ ). Black lines indicate the LES mean values, and gray area the LES interquartile range; red lines and red areas indicate the mean and the interquartile range, respectively, of the 10-member EDMF ensemble. Blue dashed lines in (a) indicate the vertical levels at which precipitation is shown.

In the SCM, the large-scale horizontal advection terms, subsidence, radiative fluxes, wind forcing, as well as the initial conditions are all prescribed. For the RICO case, the sea surface temperature (SST) and surface roughness length are both fixed and the surface fluxes are computed using the Monin-Obukhov similarity theory. For the LBA case, the prescribed time-dependent surface fluxes mimic their diurnal evolution. The prognostic equations for moist conserved variables, horizontal wind speed components, and TKE are solved on a vertically staggered grid. Updraft properties and TKE are defined on midlevels, while the other prognostic variables are defined on full levels. The vertical resolution of the SCM and the time step are $40 \mathrm{~m}$ and $40 \mathrm{~s}$, respectively.

\section{a. Precipitating marine convection}

The case of shallow precipitating convection is based on measurements collected during an undisturbed period of the RICO field campaign (Rauber et al. 2007). It represents a slowly deepening cloud layer with a steadily increasing surface precipitation. The initial and boundary conditions are taken from the LES intercomparison study by van Zanten et al. (2011). In this case, only warm microphysics is active and the mixed and ice phases are absent. We simulate the development of convection for the 24-h time period, and compare the results against the ensemble of LES models from van Zanten et al. (2011). Even though we show the interquartile range of EDMF results from 10 SCM realizations and the interquartile range from 11 LES models, we do not expect agreement between these two interquartile ranges. This is because in EDMF the spread of the results is dominated by convective variability associated with stochastic entrainment. For the LES, the spread also includes differences in numerics and physical parameterizations among the models.

\section{1) General behavior}

Figure 2a compares EDMF and LES cloud-base and cloud-top evolutions, and Figs. $2 \mathrm{~b}-\mathrm{d}$ show time series of the grid-mean cumulative rain rates at several model levels: at the surface, below the cloud base (at the height of $500 \mathrm{~m}$ ), and in the central part of the cloud layer (at the height of $1500 \mathrm{~m}$ ). Figure 3 compares the profiles of moist conserved thermodynamic variables, their corresponding subgrid vertical fluxes, TKE, and rain rates, averaged over the last simulation hour. This comparison is representative of the results for the entire simulation. For the RICO case downdrafts are relatively weak and the cold pool activity does not impact convection development. This is because the cold pool velocity $w_{D}$ [Eq. (29)] never exceeds the Deardorff surface velocity scale $w_{*}$.

Figure 2a shows that EDMF accurately simulates the vertical extent of the convective cloud layer and, in particular, its deepening with time. It should be noted that the EDMF evolution of the cloud-top height in the 

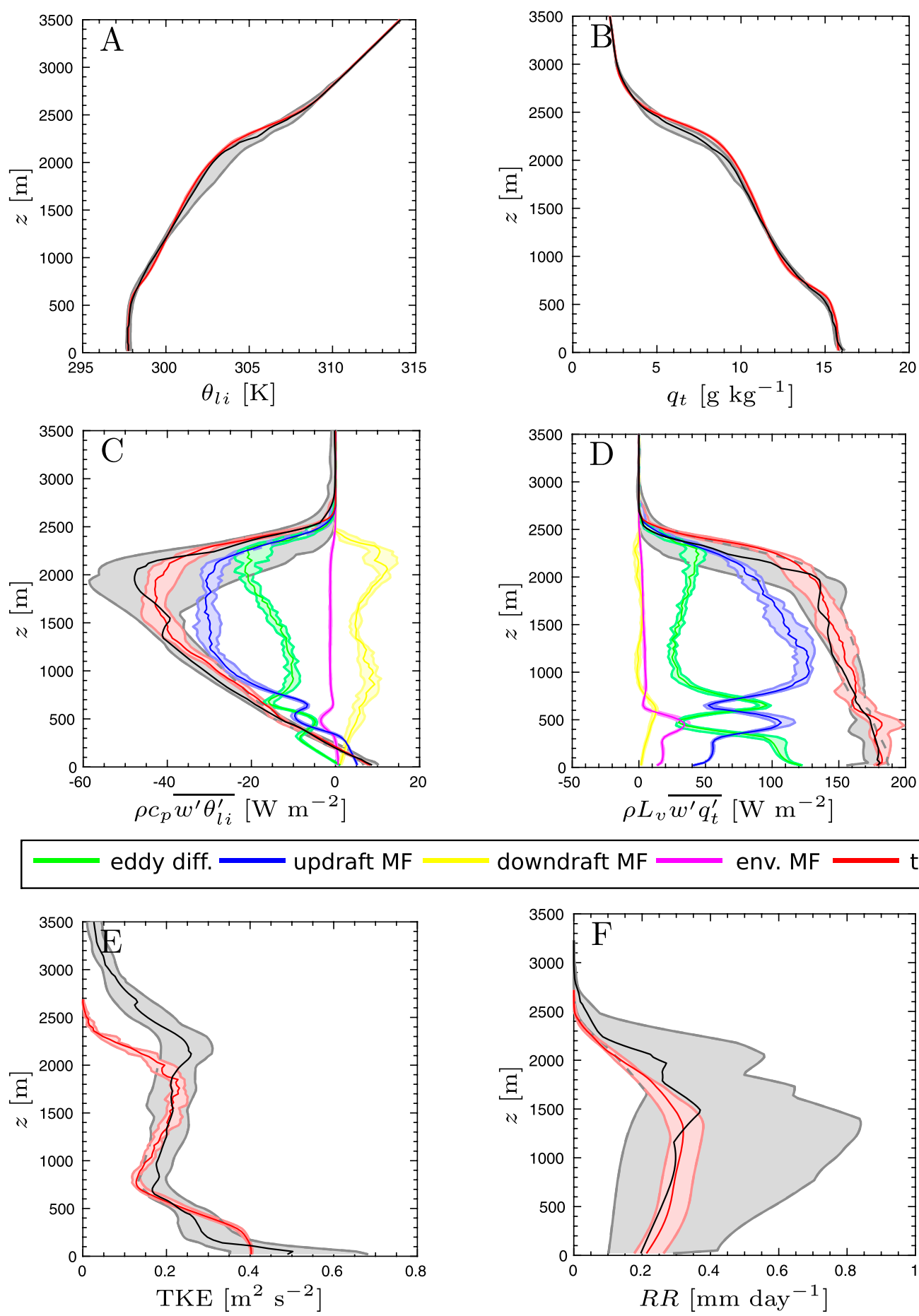

downdraft MF

env. MF

total

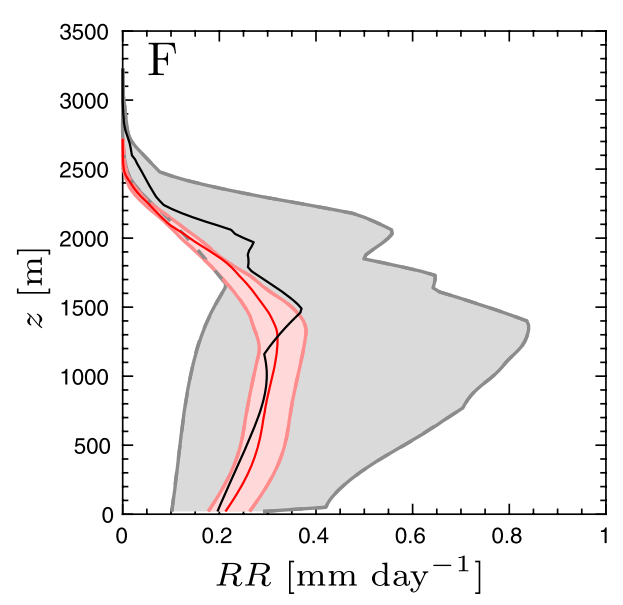

\section{EDMF}

\section{LES}

FIG. 3. RICO case. Profiles of (a),(b) mean moist conserved variables, (c),(d) their corresponding vertical subgrid fluxes, (e) TKE and (f) rain rate averaged across the last simulation hour (between hours 23 and 24). Black lines and gray area represent the median and interquartile range of the ensemble of LES results. Red lines and shaded areas represent mean and interquartile range, respectively, from the 10-member EDMF ensemble. In (c) and (d) lines and shaded areas represent the contributions to the flux from four components [see Eq. (7)]: green for eddy diffusivity, blue for updraft mass flux, yellow for downdraft mass flux, magenta for mass flux from subsiding environment, and red for the sum of all components. 
first few hours is much smoother than in LES, as the latter seems to be affected by the spinup effects. This deepening is primarily due to the rising of the cloud top and only marginally due to the lowering of the cloud base, which are both well simulated by EDMF. The consistency between the EDMF and LES results is due to similar evolutions of the moist conserved thermodynamic variables (Figs. 3a,b). The main difference regards the temperature inversion at the top of the cloud layer (i.e., around $2800 \mathrm{~m}$ ), which is around $150 \mathrm{~m}$ deeper in EDMF. The lower part of the cloud layer is slightly drier and warmer, and the upper part is moister and colder compared to LES indicating somewhat excessive vertical transport in the cloud layer. The EDMF profiles of the subgrid-scale vertical fluxes of thermodynamic variables (Figs. 3c,d) also compare well to their LES counterparts. The only significant difference is that the EDMF fluxes drop to zero somewhat higher, in agreement with the differences in the mean thermodynamic profiles. While the LES flux of total water mixing ratio is almost constant with height, the EDMF flux tends to show some variation around the cloud base. This occurs around the height where the massflux part becomes the dominant component of the turbulent flux contribution. As discussed in Suselj et al. (2019) this feature is sensitive to the parameterization of updraft vertical velocity and is present in many EDMF models. All four components of the subgrid vertical fluxes from Eq. (7) significantly contribute to the total fluxes of moist conserved variables. Both the eddy-diffusivity and the updraft mass-flux components are important throughout the whole subcloud and cloud layers. Downdrafts play an important role only for the subgrid flux of $\theta_{l i}$. The contribution from the mass flux of the environment is significant only in the upper part of the subcloud layer. Generally, one can expect that the latter becomes important only when the updraft area is relatively large. For the current setup, such conditions occur in the upper part of the subcloud layer where the updraft area is close to the surface value (i.e., $15.7 \%$ ), while the differences between the environmental and the grid-mean values of the moist conserved variables are the largest (not shown). The profiles of the subgrid vertical fluxes of moist conserved variables from the ensemble of EDMF simulations reveal a substantial variability (as measured by the interquartile range) only in the cloud layer. Overall, this variability is lower than for the ensemble of LES results, for the reasons discussed earlier. Partitioning of the subgrid vertical fluxes among the four EDMF components strongly depends on the prescribed surface updraft area (not shown), which is similar to the results discussed in Suselj et al. (2019).
The representation of TKE (Fig. 3e) has been improved compared to the earlier version of the EDMF model (Suselj et al. 2013). The key difference between the TKE from the LES and EDMF is only above the height of around $2000 \mathrm{~m}$. A detailed inspection of the TKE budget terms [Eq. (2)] reveals that in the upper cloud layer (between the heights of around 2000 and $2500 \mathrm{~m}$ ) the eddy-diffusivity contribution to the buoyancy flux ${ }^{1}$ is negative and its magnitude is overestimated compared to LES (not shown). This sink term is responsible for the excessive destruction of TKE in the upper cloud layer. Above the cloud layer, we expect that TKE in the LES is dominated by gravity waves (e.g., Tan et al. 2018), which are not represented by the EDMF model. The key to realistic modeling of EDMF TKE in the cloud layer is using a ratio between the eddydiffusivity and eddy-viscosity length scales that depends on the Richardson number (Suselj et al. 2019).

The profiles of precipitation rates (Fig. 3f) and, in particular, the evolutions of cumulative precipitation (Figs. 2b-d) from the LES ensemble feature a significant spread and thus have a limited skill in constraining EDMF. In both models, the strongest precipitation occurs well within the cloud layer (at around $1500 \mathrm{~m}$ ). In EDMF, the location of this maximum is controlled by the parameterization of rain evaporation. In the absence of evaporation, the maximum precipitation rate would be reached at the cloud base and remained unchanged throughout the subcloud layer, as it would simply be a vertical integral of the autoconversion source term from that height to the top of the model [see Eqs. (15), (18), and (26)]. The evolution of precipitation at different heights and the profile of precipitation rate from EDMF are close to the LES mean and falls well within the LES interquartile range.

To assess the representation of convective updrafts in EDMF, Fig. 4 compares the area-weighted mean moist updraft properties averaged over the last simulation hour against the cloud and cloud-core values from the LES ensemble. ${ }^{2}$ The EDMF updraft values are computed from 10 simulation runs, and the ensemble mean and the interquartile range are plotted. As in our previous work (e.g., Suselj et al. 2013, 2019), we expect the EDMF moist updraft properties to be contained within these two conditionally averaged LES profiles. Areaweighted mean dry updraft properties from the EDMF

\footnotetext{
${ }^{1}$ The buoyancy flux $g / \theta_{0} \overline{w^{\prime} \theta_{v}^{\prime}}$ comprises of the eddy-diffusivity and mass-flux components [Eq. (7)].

${ }^{2}$ Cloud grid points are defined in LES as the grid points with positive liquid water, whereas cloud-core grid points also need to be positively buoyant with respect to the slab average.
} 

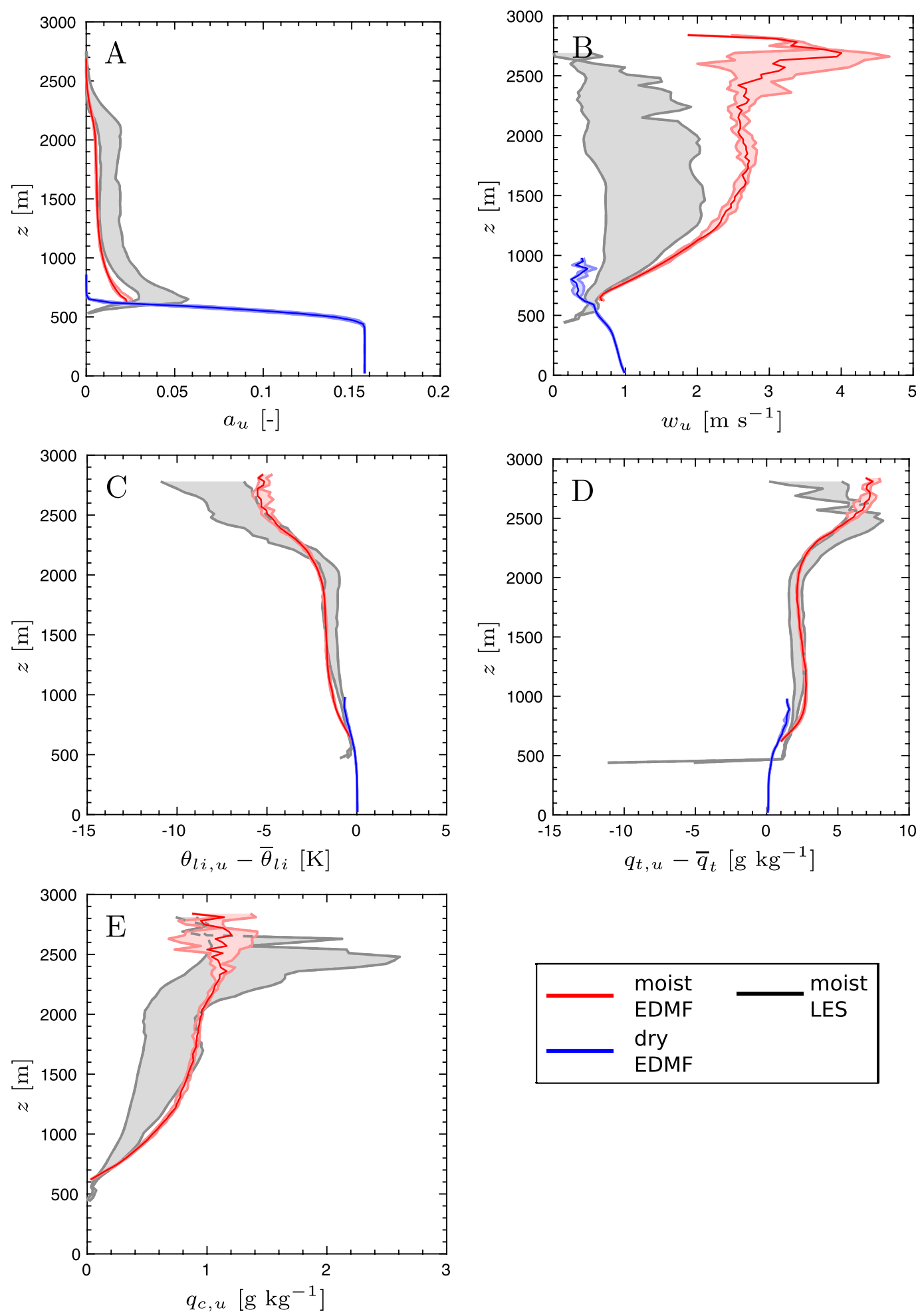

FIG. 4. RICO case. Profiles of (a) moist updraft area, (b) updraft vertical velocity, (c),(d) difference of the moist conserved variables from updrafts with respect to the mean moist conserved variables, and (e) updraft cloud water averaged across the last simulation hour. Gray area encompasses the LES cloud and cloud-core values. Red lines are for area-weighted mean moist updrafts from EDMF, and blue lines are for area-weighted mean dry updrafts from EDMF. Red and blue areas represent interquartile ranges computed from 10 EDMF ensemble members for moist and dry updrafts, respectively. 
are also shown, although we do not have a relevant reference from the LES model.

The EDMF moist updraft properties agree reasonably well with the LES results. The main differences regard the updraft vertical velocity and the fractional updraft area. While the former tends to be overestimated by EDMF, especially in the upper part of the cloud layer, the latter is slightly lower than the cloud-core profile between 1500 and $2000 \mathrm{~m}$. These differences are less important for the vertical fluxes due to updrafts, which are proportional to their product. Figure 4a shows that essentially all of the updrafts survive up to the cloud base. At the cloud base most of the updrafts terminate and only a small fraction condenses and continues rising as moist updrafts, which gradually terminate as they rise. The dry fractional updraft area at the surface is in this model fixed at $15.7 \%$ while the fractional area of moist updrafts at the cloud base is approximately $2 \%$. A small number of moist updrafts reach high enough to form precipitation. The maximum fractional area of precipitating updrafts is approximately $0.35 \%$ (not shown). In the subcloud layer, the vertical velocity of dry updrafts and the excesses of moist thermodynamic variables from the mean values are relatively small compared to the moist updrafts. For the RICO case, the EDMF model simultaneously represents dry convection, and moist nonprecipitating and precipitating convection.

To understand why the updrafts terminate at different heights, Figs. $5 \mathrm{a}$ and $5 \mathrm{~b}$ show the PDFs of the surface updraft index and the vertically averaged entrainment rates computed for the updrafts that survive to a certain height. This figure shows statistics for three representative heights: just below the cloud base $(500 \mathrm{~m})$, above the cloud base $(600 \mathrm{~m})$, and for the upper cloud layer $(2300 \mathrm{~m})$. While almost all surface updrafts reach the height of $500 \mathrm{~m}$, around $50 \%$ of them reach the height of $600 \mathrm{~m}$ and only $2 \%$ of the updrafts reach the highest studied level.

As in Suselj et al. (2019), the updraft index is defined as $n=1, \ldots, N$ (see section $2 \mathrm{~d}$ ) to number the bins of the surface PDFs used to initialize the updrafts. Therefore, updrafts with higher surface indices are characterized by higher near-surface vertical velocity, buoyancy, and moisture.

The surface updraft index distributions are uniform at the surface (by definition) and just below the cloud base. These results are consistent with a constant updraft fractional area in that layer (Fig. 4a), which indicate that there is no termination of updrafts within the well-mixed layer. The updrafts with both lower average entrainment rate and higher surface updraft index are more likely to penetrate higher through the cloud base to reach $600 \mathrm{~m}$. This is seen as a shift of the vertically averaged entrainment rates toward smaller values and a shift of the surface updraft index toward larger values in the layer between 500 and $600 \mathrm{~m}$. Once the updrafts condense and survive to the lower part of the cloud layer, their termination height becomes primarily dependent on their entrainment rate as there is little change in the PDF of the updraft surface index between 600 and $2300 \mathrm{~m}$. At the same time, the vertically averaged entrainment rates shift toward smaller values between these two heights indicating that the updrafts associated with smaller entrainment rates reach higher in the cloud layer. Figures $5 \mathrm{c}$ and $5 \mathrm{~d}$ visualize this behavior by showing the vertical velocity of individual updrafts from five snapshots of one of the EDMF realizations, color coded by their updraft index (Fig. 5c) and vertically averaged entrainment rates (Fig. 5d). This figure provides further evidence that most of the updrafts terminate around cloud base, while only the ones with higher surface index and smaller entrainment rate penetrate into the cloud layer. Above the cloud layer, their termination height is mostly controlled by the entrainment rate. These results agree with the findings for the shallow nonprecipitating convection case discussed in Suselj et al. (2019).

Figure 6 shows the area-weighted downdraft properties averaged over the last simulation hour from 10 EDMF realizations. A reference dataset from LES is not shown as it is not available. The downdraft area increases from the cloud-top height to approximately $1300 \mathrm{~m}$ above the surface, and stays constant between that level and the surface. This is because for each precipitating updraft a complimentary downdraft is initiated, and because precipitating updrafts gradually terminate between the height of $1300 \mathrm{~m}$ and the overall cloud top. Once the downdraft is initiated it extends from the initiation height to the surface. The magnitude of downdraft velocity is the highest in the upper part of the cloud layer where they can be much colder than the environment. The evaporation of precipitation provides strong cooling of the downdrafts in this layer. Overall, the downdrafts tend to be colder than the environment throughout the cloud and subcloud layers. The total water mixing ratio is close to the environmental value in the cloud layer. The differences between the downdraft and gridmean moist conserved variables peak in the upper part of the subcloud layer, where the vertical gradients of the grid-mean values are the highest. As the downdrafts penetrate deeper into that layer, the differences reduce because of lateral entrainment and rain evaporation. 

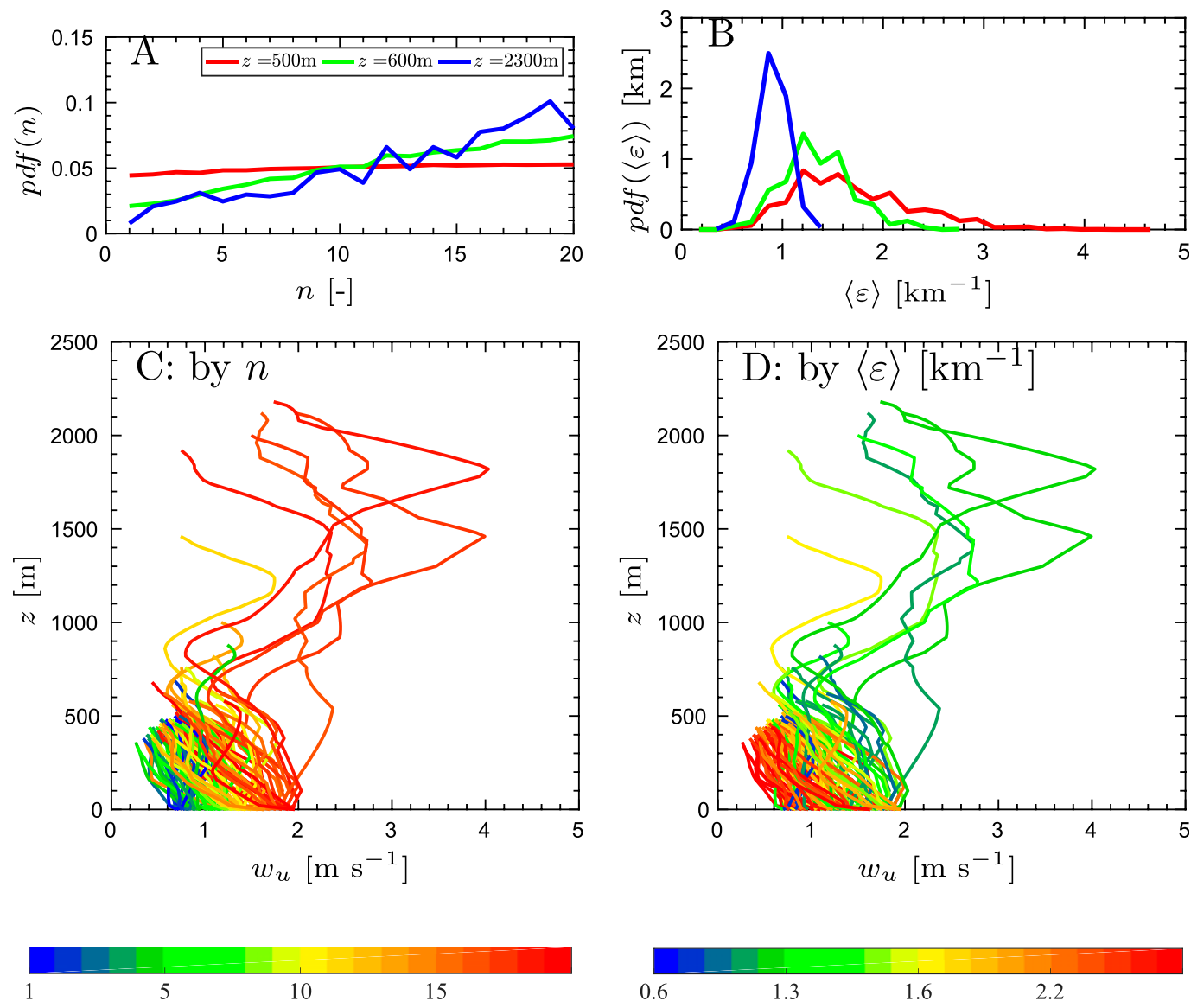

FIG. 5. RICO case. Probability density of (a) updraft index and (b) vertically averaged entrainment rate for updrafts that reach the height of just below cloud base (height of $500 \mathrm{~m}$; red line), just above the cloud base (height of $600 \mathrm{~m}$; green line), and upper cloud layer (height of $2300 \mathrm{~m}$; blue line). Probability density is normalized so that its integral over the range of the values defined by $x$ axis equals unity. Statistics are computed from 10 EDMF realizations for instantaneous updrafts within the last simulation hour. (c),(d) The vertical velocity of individual updrafts from five snapshots from one of the simulations. The updrafts are color coded by their surface index in (c) and vertically averaged entrainment rate in (d).

\section{2) ROLE OF MICROPHYSICAL PROCESSES FOR CONVECTION DEVELOPMENT}

To understand how the microphysical processes modify the thermodynamic structure of the troposphere, Fig. 7 shows the differences in the profiles of moist conserved variables between the default RICO experiment and two modified experiments with a reduced impact of microphysics. The modified experiments either disable evaporation of precipitation [referred to as RICOne; $k_{e}$ is set to zero in Eqs. (17) and (23)] or completely disable precipitation formation [RICOnp; $S_{q_{t}}^{\text {ac }}$ is set to zero in Eq. (15)]. We show the results from the RICOne to argue that the evaporation of precipitation is a key microphysical process that modifies the profiles of updraft structure and explains a large part of the differences between the RICO and RICOnp results. Figure 7 also includes the difference between RICO and RICOnp experiments from the LES ensemble from van Zanten et al. (2011).

In the LES experiments the absence of precipitation promotes a deeper cloud layer. By the end of the simulation, the cloud layer in RICOnp is approximately $100 \mathrm{~m}$ deeper compared to the cloud layer in the RICO experiment (van Zanten et al. 2011). Similar results are obtained for the SCM (not shown). The differences in the cloud-top height between these two experiments are reflected by a positive anomaly of $\theta_{l i}$ (up to $2 \mathrm{~K}$ ) and a negative anomaly of $q_{t}$ (up to $-1.5 \mathrm{~g} \mathrm{~kg}^{-1}$ ) at around $2500 \mathrm{~m}$ (Fig. 7). In the EDMF simulations, the peak of this anomaly is located somewhat higher compared to the LES ensemble because of a slightly higher inversion height. Nevertheless, the magnitude of the differences between the RICO and 

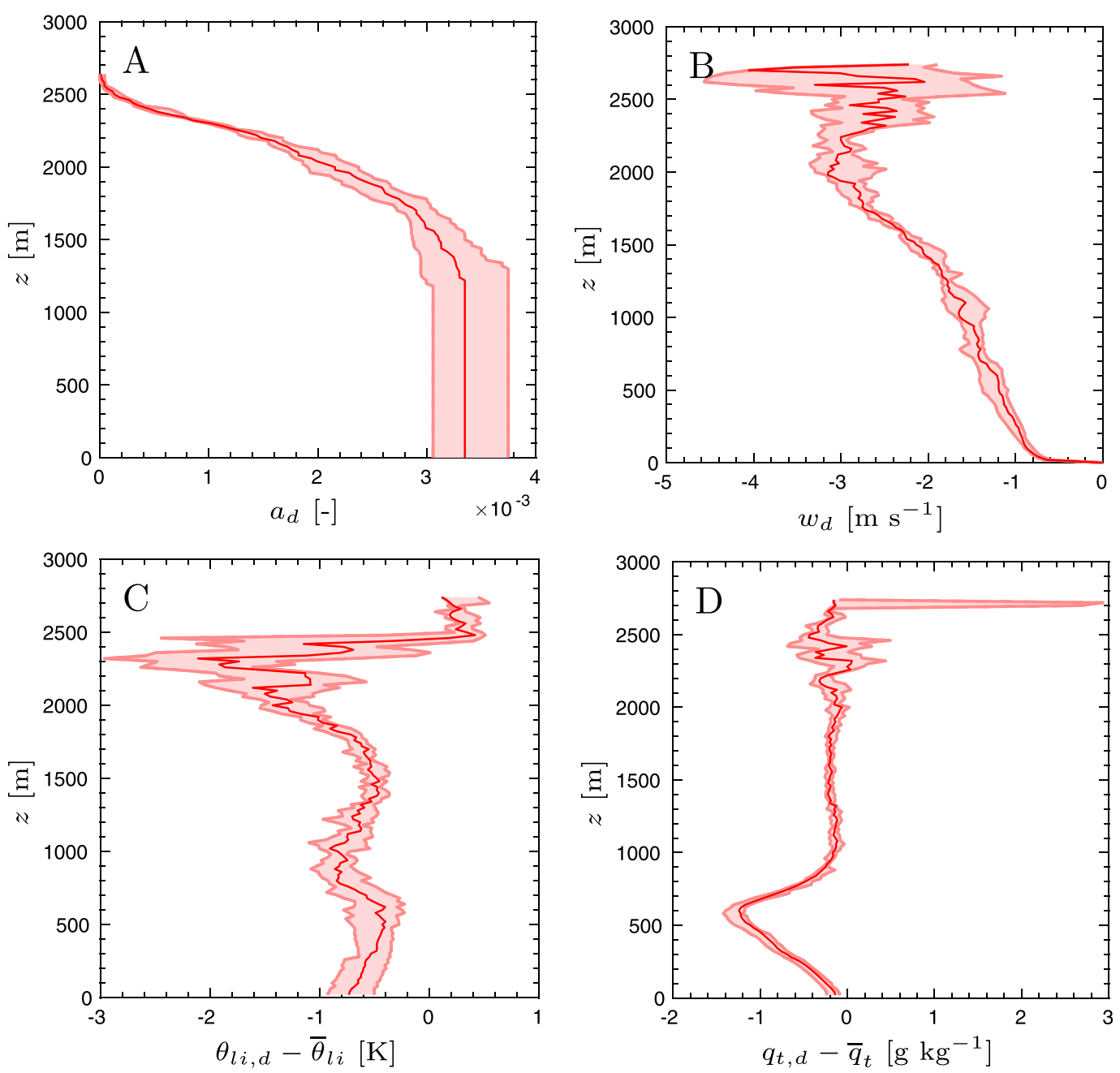

FIG. 6. RICO case. Profiles of (a) downdraft area, (b) vertical velocity, and (c),(d) difference of the moist conserved variables from downdrafts with respect to the mean moist conserved variables. Red lines and red areas represent the mean and interquartile range, respectively, of the 10-member EDMF ensemble.

RICOnp experiments from EDMF agrees reasonably well with the LES results. Note that the EDMF results are the closest to the LES results when the precipitation effects are the largest and the differences between the RICO and RICOnp experiments are the largest too. These results indicate that the impact of precipitation formation on the depth of the cloud layer is well captured by the EDMF model.

The EDMF subcloud layer and most of the cloud layer are both slightly moister and colder in RICO than in RICOnp. While the moisture differences agree well with LES, the temperature differences are around $0.2 \mathrm{~K}$ lower, which increases the cloud-layer depth. These differences are dominated by the evaporation of precipitation since they are similar to those between the RICO and RICOne experiments (Fig. 7). The only substantial difference between the RICOnp and RICOne results regards the upper part of the cloud layer, where the RICOnp profiles are notably colder and moister. This is linked with stronger updrafts in RICOnp presumably because precipitation formation reduces the buoyancy of the plumes for the default case. This comparison shows that in the EDMF model the evaporation of precipitation is indeed the microphysical process that most significantly impacts thermodynamic structure in the precipitating RICO case.

\section{3) SEnSitivity to THE NUMBER OF UPDRAFts}

To assess the sensitivity of the results to the number of updrafts $N$ per time step, Fig. 8 shows cumulative surface precipitation at $24 \mathrm{~h}$ and its spread as a function of $N$. Each EDMF ensemble consists of 10 simulations. The cumulative surface precipitation is 

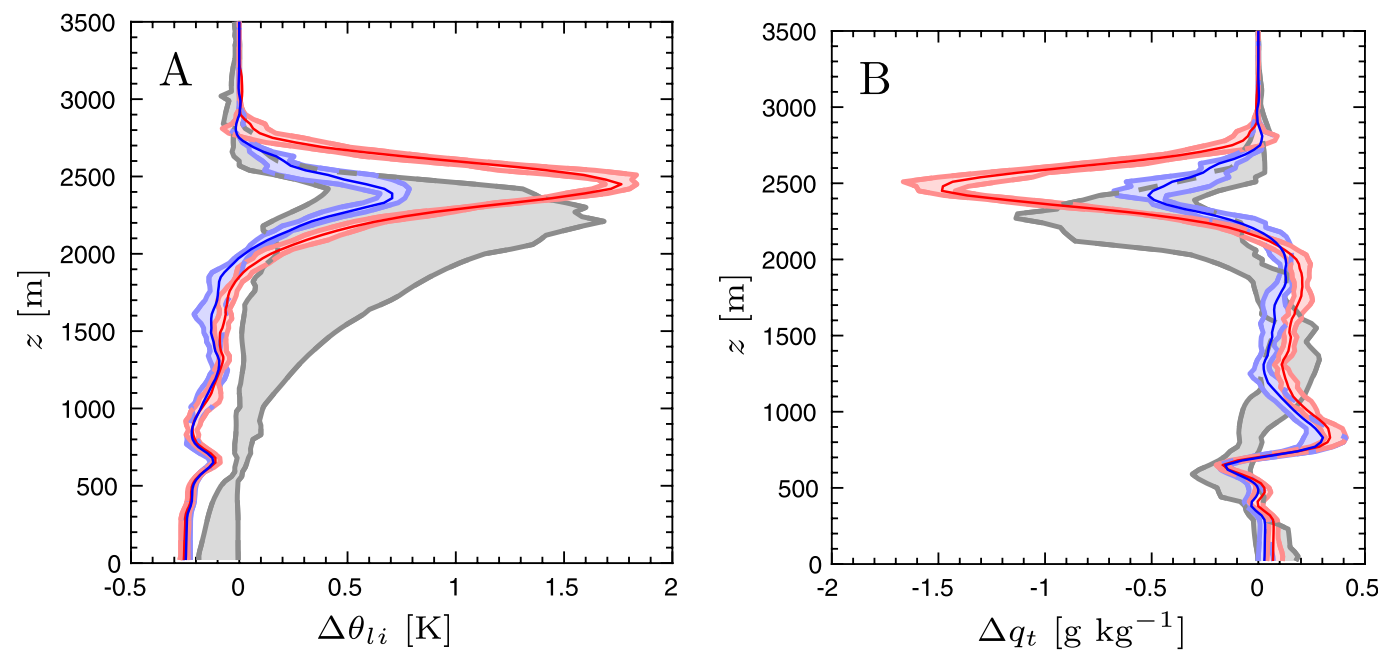

LES: RICO-RICOnp EDMF: RICO-RICOnp

EDMF: RICO-RICOne

FIG. 7. RICO case. Differences in the thermodynamic profiles of (a) $\theta_{l i}$ and (b) $q_{t}$ between the LES precipitating (RICO) and nonprecipitating (RICOnp) cases (black lines), EDMF RICO and RICOnp cases (red lines), and EDMF RICO and nonevaporating (RICOne) cases (blue lines). Shaded areas represent the interquartile ranges from the ensemble of simulations.

shown because it is one of the results that is most sensitive to parameter $N$.

The results show that the ensemble cumulative precipitation generally increases with the number of updrafts. The precipitation is most sensitive to parameter $N$ when its value is less than 10 . Once the number of updrafts exceeds that value, cumulative surface precipitation only marginally increases. It is likely that this sensitivity is a result of nonlinearity of the rain production and evaporation within individual updrafts that is better resolved with a larger set of plumes. Figure 8 also shows that the variability of cumulative precipitation strongly decreases with the number of updrafts when their number is smaller than 10 and stays fairly constant for larger numbers.

\section{b. Diurnal cycle of continental convection}

The case representing the diurnal cycle of continental convection is based on measurements performed during the LBA experiment in Amazonia. Convection development is forced by the time-dependent surface sensible and latent heat fluxes. The simulation starts from a shallow dry and well-mixed boundary layer. As time progresses, the dry boundary layer gradually deepens and transitions to moist shallow and then to deep heavily precipitating convection.

The simulation setup closely follows Grabowski et al. (2006) with modifications described in Kurowski et al. (2018). The prescribed surface fluxes start from zero (at 0730 local time), peak $5.25 \mathrm{~h}$ later (at 1245 local time), and decrease in the later hours so that they are symmetric around the peak time. The radiative forcing is set to zero, which differs from Grabowski et al. (2006), who prescribe a relatively weak radiative cooling, which has however no major impact on convection development.

The reference results are taken primarily from Kurowski et al. (2018) and are obtained using the LES version of the Weather Research and Forecasting (WRF) Model (Skamarock et al. 2008). The horizontal domain covers an area of $70 \mathrm{~km} \times 70 \mathrm{~km}$. The domain extends vertically from the surface up to $21 \mathrm{~km}$, which includes a sponge layer applied to the layer above $17 \mathrm{~km}$ to suppress gravity wave reflections from the rigid-lid upper boundary. The domain is laterally doubly periodic with a uniform horizontal

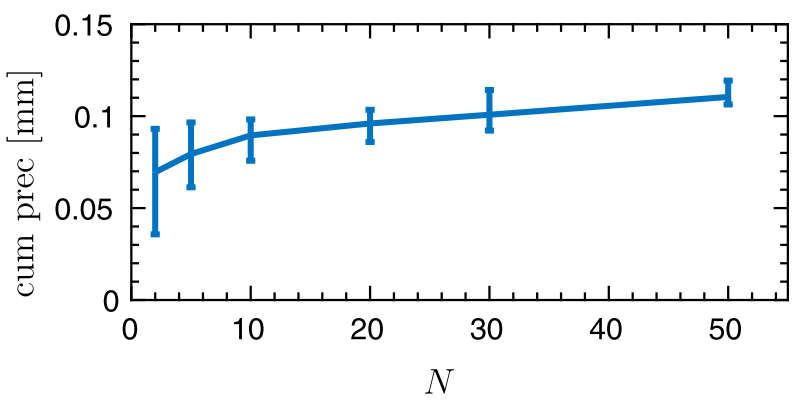

FIG. 8. RICO case. Sensitivity of cumulative precipitation (cum prec) to the number of updrafts $N$. The error bars represent the range of precipitation from the ensembles of 10 simulations each. 
grid spacing of $100 \mathrm{~m}$. The model applies 128 vertical levels on a stretched grid, with the grid length increasing from around $60 \mathrm{~m}$ near the surface to around $300 \mathrm{~m}$ near the domain top. The subgrid-scale turbulence closure is based on a prognostic equation for turbulent kinetic energy (e.g., Deardorff 1980). Three different reference simulations are performed. One simulation uses a complete microphysical scheme (which we refer to as the LBA case) and is equivalent to the CP_p experiment in Kurowski et al. (2018). In this simulation, the microphysics is modeled using a single-moment Lin-Purdue scheme (Lin et al. 1983) with six classes of water species (i.e., water vapor, cloud liquid water, rain, ice, snow, and graupel). In the second simulation, cold pools are disabled by horizontally averaging the rain-evaporative tendencies. This simulation is the same as NOCP_p in Kurowski et al. (2018) and we will here refer to it as LBAnocp. The third simulation represents a simplified warm nonprecipitating version of the LBA case (LBAnp), where microphysical processes are disabled and only condensation and evaporation with respect to the liquid phase are enabled.

Because microphysical processes significantly impact the development of deep convection and the thermodynamic structure of the atmosphere, we first analyze the behavior of the EDMF model for the warm nonprecipitating LBA case (LBAnp). To understand the role of microphysical processes, we then focus on the precipitating LBA case. The impact of cold pools is evaluated by comparing the results from the LBA and LBAnocp cases.

\section{1) WARM NONPRECIPITATING LBA CASE}

Figure 9 compares the evolution of the cloud-base and cloud-top heights from the LES and EDMF models, for the LBAnp case. In LES, the cloud layer starts forming about $1 \mathrm{~h}$ into the simulation. As time progresses, the layer gradually deepens, primarily because of the increasing cloud-top height. Concurrently, the cloud-base height gradually rises but at a much lower rate. The most rapid growth occurs between hours 2 and 5. After the surface fluxes peak at hour 5.25, the growth of the cloud layer ceases almost completely. Generally, the EDMF evolution agrees well with that of LES. In EDMF, the growth of cloud layer in the morning hours is dominated by the deepening of the moist convective layer (i.e., of moist updrafts) while its persistence in the afternoon hours is due to the cloud formation in the nonconvective environment (not shown). The rapid growth of the convective layer partially results from a positive feedback between the depth of convective updrafts

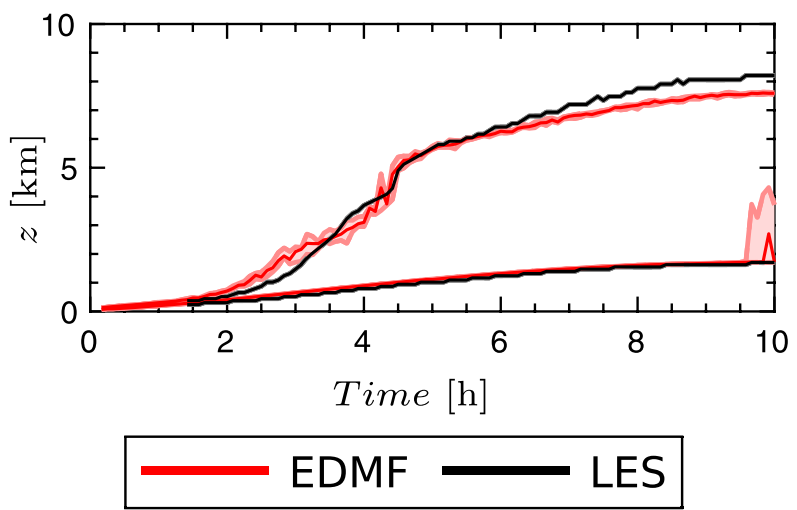

FIG. 9. LBAnp case. Time series of the cloud-base and cloud-top heights. Black and red lines represent the LES and EDMF results, respectively. Black and red shadings are the interquartile ranges from the LES and EDMF ensembles, respectively.

and the entrainment rate [Eq. (30)]. As the convective layer deepens, the mean entrainment rate decreases, which promotes further deepening of the convection. There are also some noticeable differences between the LES and the EDMF results. In EDMF, shallow convection sets in almost at the beginning of the simulation and the cloud layer tends to be somewhat deeper during the first three simulation hours and shallower for the last part of the simulation. Figure 9 also shows that the rapid-growth phase is characterized by the largest spread in the EDMF results.

Figure 10 compares hourly averaged profiles of the moist conserved variables and their vertical subgrid fluxes from EDMF and LES, and Fig. 11 compares the profiles of cloud water mixing ratio, cloud cover, and moist static energy (MSE). ${ }^{3}$ The results from the early (second simulation hour), intermediate (fourth hour), and mature (seventh hour) stages of convection development are shown. Clearly, the two models yield consistent gradual warming and drying of the subcloud layer that remains relatively well mixed throughout the simulation. In EDMF, the drying is primarily due to a strong transport of moisture from PBL into the cloud layer by convective updrafts that exceeds the moistening due to surface fluxes (not shown). At the same time, the cloud layer aloft gradually moistens and cools over the course of the simulation. The cooling/moistening intensifies in the last several hours in the upper part of the cloud layer (between 4 and $7 \mathrm{~km}$ ), which becomes an accumulation zone for the condensed water associated

\footnotetext{
${ }^{3}$ Moist static energy is defined as MSE $=c_{p} T+g z+L_{v} q_{v}$, which is the sum of sensible, potential, and latent enthalpy. For the purpose of this analysis, we normalize it by $c_{p}$.
} 

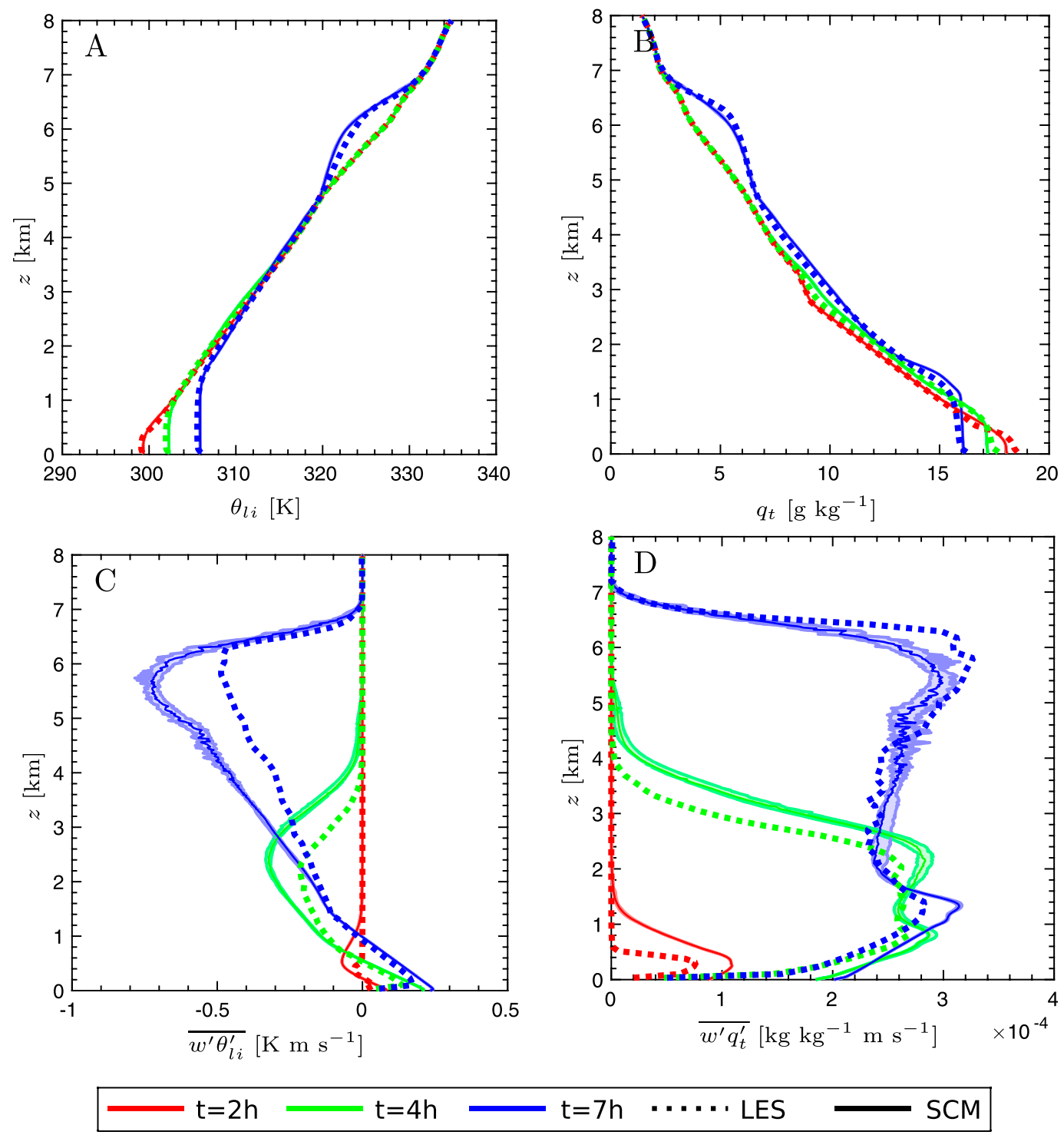

FIG. 10. LBAnp case. Profiles of hourly averaged moist conserved variables: (a) $\theta_{l i}$, (b) $q_{t}$, and (c), (d) vertical subgrid fluxes of the moist conserved variables, at hours 2 (red), 4 (yellow), and 7 (blue). The dashed and solid lines represent the LES and EDMF results, respectively. Shaded areas show the interquartile range of the 10-member EDMF ensemble. The LES subgrid fluxes in (c) and (d) include only the resolved component.

with overcast conditions, capped by a strong temperature inversion.

Profiles of the vertical subgrid fluxes of the moist conserved variables agree well between EDMF and LES, which is the key reason for the consistent evolution of the thermodynamic properties. As already seen in Fig. 9, EDMF simulates an earlier onset of convection that remains deeper for the first part of the simulation, which here corresponds to a somewhat larger magnitude of the subgrid fluxes in the second hour, with the vertical transport reaching around $1 \mathrm{~km}$ higher than in LES. This difference persists for the next $2 \mathrm{~h}$ and diminishes at the mature stage of convection. Arguably, the LES solution tends to be delayed because of the lack of a fully developed cascade of turbulent eddies in the first hour, meaning that the parameterization evaluation should be treated with caution when comparing the elements that are fundamentally different. As for the RICO case, the subcloud mixing in EDMF is dominated by the combination of eddy diffusivity, mass flux due to convective updrafts, and mass flux from the subsiding environment, while the transport in the cloud layer is dominated by the mass flux from convective updrafts 

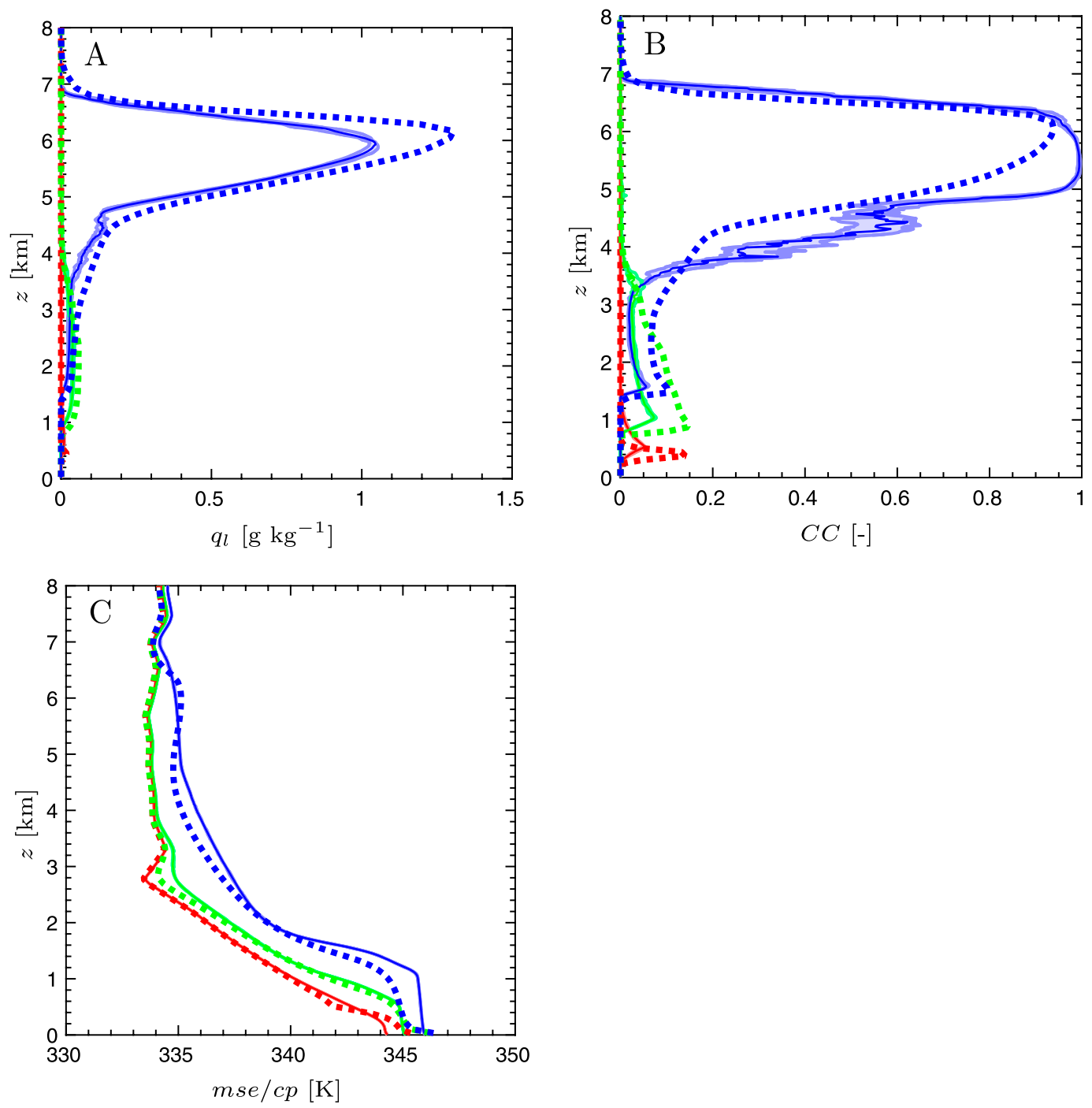

$$
\mathrm{t}=2 \mathrm{~h} \longrightarrow \mathrm{t}=4 \mathrm{~h} \longrightarrow \mathrm{t}=7 \mathrm{~h} \quad \cdots \mathrm{LES} \longrightarrow \mathrm{SCM}
$$

FIG. 11. LBAnp case. Profiles of hourly averaged (a) cloud water mixing ratio, (b) cloud cover, and (c) moist static energy, at hours 2 (red), 4 (yellow), and 7 (blue). The dashed and solid lines represent the LES and EDMF results, respectively. Shaded areas show the interquartile range of the 10-member EDMF ensemble.

(not shown). An exception is the increased contribution from the environmental turbulence in the upper cloudy region (between 4 and $7 \mathrm{~km}$ for the seventh hour), which is a consequence of the increased TKE in this layer because of the enhanced buoyancy production from condensation (not shown). The evolution of the cloud cover and the cloud water mixing ratio from EDMF are both in good agreement with LES (Figs. 11a,b). For the two models, cloud cover is small in the first several hours (for EDMF, condensation only occurs within convective updrafts), with overcast conditions developing later in the day in the upper layers (associated with stratiform cloud formation in the environment).

The evolution of MSE (Fig. 11c) summarizes the evolution of moist conserved variables. The sources of MSE are prescribed at the surface through the sensible and latent heat fluxes, and the role of convection is to transport MSE from the surface up to higher levels. Figure 11c shows a monotonic increase of MSE in the subcloud and cloud layers throughout the day. In the subcloud layer, the MSE increase is primarily due to the temperature increase, whereas in the cloud layer it is primarily due to the moisture increase. The process 

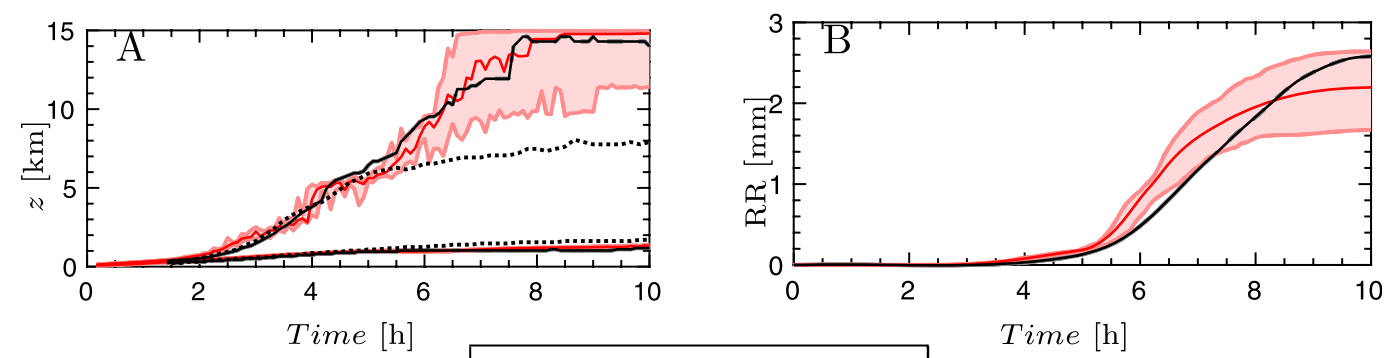

EDMF LES
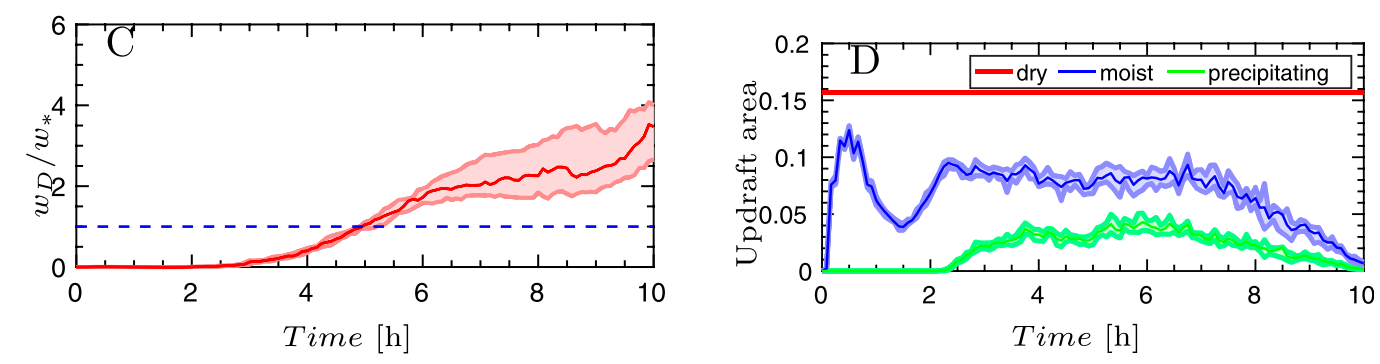

FIG. 12. LBA case. Time series of (a) cloud-base and cloud-top heights, (b) cumulative surface precipitation, (c) ratio of the velocity scales $w_{D}$ and $w_{*}$, and (d) maximum fraction of the updrafts: dry (red), moist nonprecipitating (blue), and moist-precipitating (green). In (a)-(c) black and red solid lines denote the LES and EDMF results, respectively. In (a) the dashed lines are for the LBAnp case.

of moistening the near-subcloud layer lasts for the first few hours. As the convective layer deepens, the net change in that region becomes negative at the cost of moistening the cloud layer above. Along with gradual warming, both of these effects lead to a rise in the condensation level. The MSE from EDMF agrees well with LES, except that the subcloud layer is slightly deeper and too well mixed, especially at the beginning of the simulation.

\section{2) Precipitating LBA case}

Figure 12a shows the evolution of the cloud-base and cloud-top heights from the LBA experiment, and Fig. $12 \mathrm{~b}$ the cumulative surface precipitation, from the ensemble of the EDMF simulation and LES. In Fig. 12a the results from the LBAnp case are shown as well. Figure $12 \mathrm{c}$ shows the ratio of velocity scales $w_{D}$ and $w_{*}$ from EDMF, as a measure of the strength of convection invigoration by cold pools. Note that cold pools affect convection when this ratio exceeds unity [see Eqs. (30) and (31)]. Figure $12 \mathrm{~d}$ shows the time series of maximum (over the vertical dimension) fraction of the dry, moist nonprecipitating, and moist precipitating updrafts.

For the LES, the early development of convection follows that of the LBAnp, with the first noticeable differences appearing just after the fourth hour when a significant amount of precipitation reaches the surface. Unlike the LBAnp, in which the cloud-top height saturates around the fifth hour, the deepening of convection continues at a similar rate until the eighth hour to reach $14 \mathrm{~km}$. The strongest precipitation rate occurs between the sixth and eighth hours, as indicated by the largest temporal gradient of cumulative precipitation. Consistent with the LBAnp case, the onset of moist convection is earlier in the EDMF model, with a somewhat deeper cloud layer during the first $4 \mathrm{~h}$. However, the deepening slows down in the next $2 \mathrm{~h}$ and the layer becomes slightly shallower compared to the LES around the fifth hour. After this time, convection in EDMF gradually reinvigorates because of the cold pool effects, and the cloud-top height eventually reaches $15 \mathrm{~km}$ by the end of the simulation. Figure 12c shows that in EDMF cold pools start invigorating convection around the fifth hour (when the ratio of the velocity scales exceeds unity) and this effect intensifies toward the end of the simulation. By that time, the mean ratio of the velocity scales approximately reaches the value of three. In the model, this ratio determines the cold pool-induced modifications of the entrainment rate for the updrafts and downdrafts, as dictated by Eq. (30), and the variances of the near-surface thermodynamic variables [Eqs. (31)(34)]. In the later hours, the high variability of the cloudtop height among the EDMF simulations is partially due to the stratiform cloudiness variability resulting from the differences in the advection of moisture from lower levels.

For the cumulative surface precipitation, the EDMF and LES results agree fairly well with each other in 
terms of both the amount and the timing. Consistent with the deeper cloud layer in EDMF at the initial stage of convection, surface precipitation is also stronger at that time. However, the comparison should be treated with caution since the LES results are generally strongly sensitive to the details of microphysics (e.g., Grabowski et al. 2006), with the spread expected to be even larger than for the RICO case because of stronger dynamical feedbacks. At the end of the simulation, the ensemble-mean cumulative precipitation from EDMF is approximately $2.1 \mathrm{~mm}$, which is around $0.5 \mathrm{~mm}$ less than from LES, with considerable spread of the interquartile range of $1 \mathrm{~mm}$.

Figure $12 \mathrm{~d}$ shows that the maximum of dry updraft area in EDMF is (by design) $15.7 \%$ throughout the simulation. As for the RICO case, most of the dry updrafts reach the cloud base where a fraction of them terminates while the other condense and continue rising as moist updrafts. The maximum moist updraft area peaks in the first simulation hour around $12 \%$, decreasing to $4 \%$ in the next hour and stabilizes around $8 \%$ between the third and the seventh hours. The decrease of the updraft area in the last $3 \mathrm{~h}$ is likely caused by a rapid decrease of the surface latent and sensible heat fluxes. Precipitation in the updrafts starts forming around $2.5 \mathrm{~h}$ into the simulation with sufficient deepening of moist convective clouds afterward. Between the fourth and the seventh simulation hours, moist precipitating updrafts account for approximately $4 \%$ of the total area, decreasing in later hours as the area of nonprecipitating moist updrafts decreases too.

The hourly averaged profiles of moist conserved variables (in the lowest $4 \mathrm{~km}$ ) and their vertical subgrid fluxes (in the entire troposphere) at the early (second hour), intermediate (fourth hour), and mature (seventh hour) stages of convection from EDMF and LES are compared in Figs. 13a-d. Figures 13e and 13f show the EDMF decomposition of subgrid fluxes into the four components for the seventh hour. The profiles of subgrid fluxes are similar to those from the LBAnp case, except that they penetrate deeper into the troposphere. In addition, they do not show a peak in the upper cloud layer because of the lack of extensive cloudiness (see below). Similarly to the LBAnp case, the subcloud layer gets warmer and drier over the course of the simulation. However, this process is now suppressed because of precipitation formation and its feedback on the dynamics. At the seventh hour, the near-surface layer is around $2 \mathrm{~K}$ colder and $2 \mathrm{~g} \mathrm{~kg}^{-1}$ moister than in the LBAnp case. In addition, the subcloud layer is not that well mixed, which is particularly noticeable for the vertical profile of total water mixing ratio. Overall, the LES and EDMF results generally agree well with each other in terms of the evolution of the lower-tropospheric moist conserved variables and their fluxes. One noticeable difference is that the EDMF subcloud layer is somewhat too dry and too well mixed, which is consistent with an excessive mixing in the subcloud layer. This mixing is indicated by a $10 \%-$ $20 \%$ overestimation of the vertical subgrid flux of the total-water mixing ratio in that region. Another important difference is that the flux of ice-liquid water potential temperature is notably overestimated above $5 \mathrm{~km}$ at hour 7 .

Figures $13 \mathrm{e}$ and $13 \mathrm{f}$ show that the nonlocal transport by the updrafts is the main contribution to the subgrid fluxes of moist conserved variables throughout the subcloud and cloud layers. It also controls the variability of total fluxes among the ensemble members. This variability is likely dominated by the differences in interactions between the convective updrafts and cold pools. Concomitantly, the eddy-diffusivity part is an important component of total fluxes not only in the subcloud layer but also in the cloud layer. The massflux part due to the downdrafts is most significant close to the cloud base and in the subcloud layer, while the mass flux from the environment is important only in the subcloud layer.

A comparison of the hourly averaged profiles of cloud water mixing ratio (liquid and ice combined), cloud cover, MSE, and rain rate is shown in Fig. 14. In contrast to the LBAnp, here the overcast conditions do not develop. Apparently, microphysical processes efficiently remove the moisture from the upper troposphere (transported there by convective updrafts), which prevents the formation of extensive clouds. For the three profiles shown, the mean cloud cover in the LES never exceeds $10 \%$. The profiles of cloud water mixing ratio are fairly well reproduced by the EDMF model. One notable difference is some underestimation of cloud water between 3 and $6 \mathrm{~km}$ at the seventh hour. Cloud cover is well simulated for the first two profiles but is notably underestimated in the lower troposphere and overestimated aloft at the seventh hour. In the midtroposphere, most of the cloudiness in the EDMF is due to the condensation within convective updrafts, with an insignificant contribution from the nonconvective environment. In the upper part, stratiform cloudiness in the nonconvective environment is a predominant component. After the seventh hour, the nonconvective cloudiness develops in the EDMF but not in LES (not shown). Arguably, these differences between EDMF and LES are due to the missing representation of stratiform microphysics in the EDMF parameterization. The differences in the subgrid mixing between LES and EDMF are also reflected in the differences of the MSE profiles. 

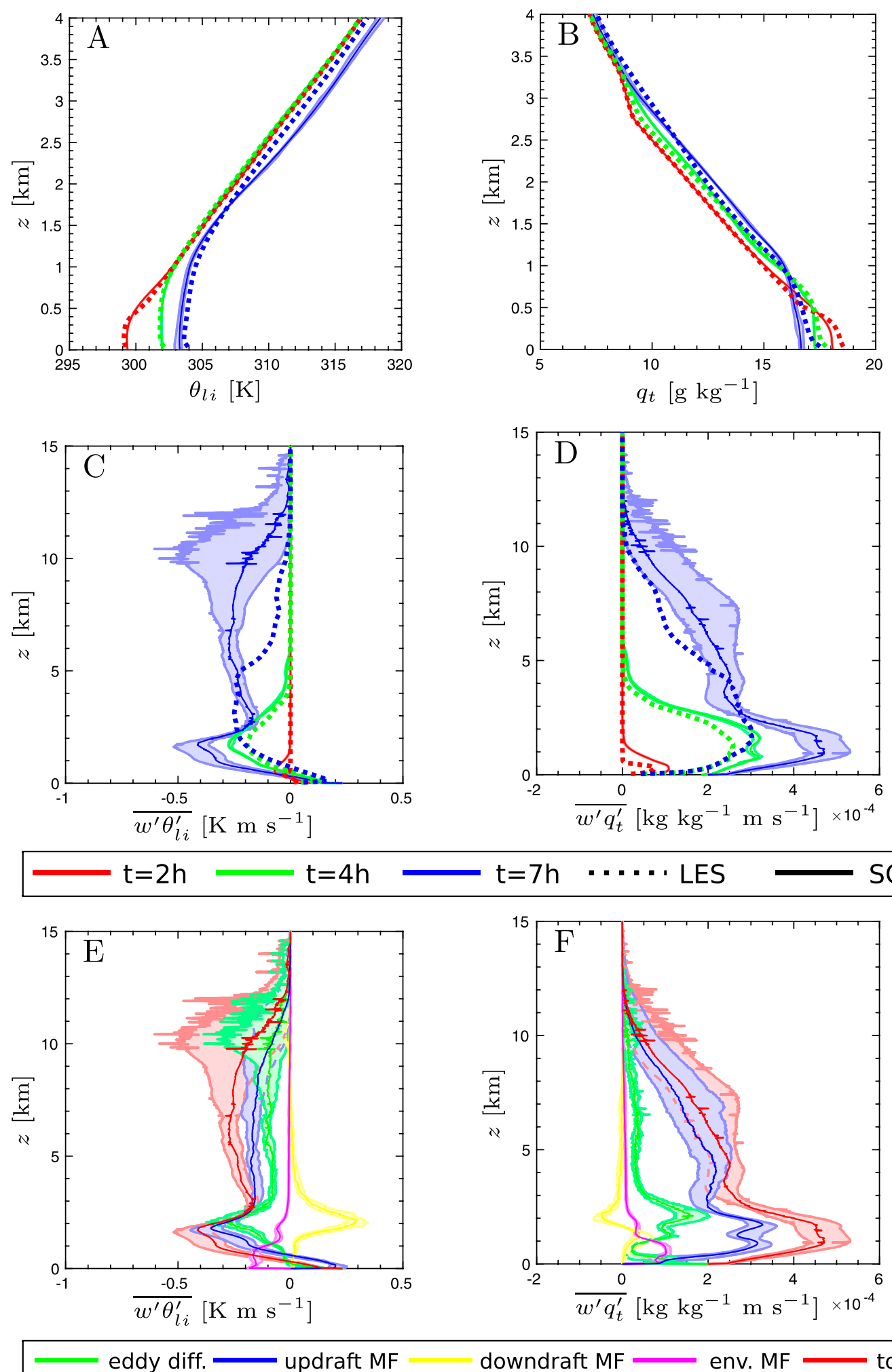

downdraft MF

env. MF

total

FIG. 13. LBA case. Profiles of hourly averaged moist conserved variables: (a) $\theta_{l i}$ and (b) $q_{t}$ in the lower troposphere, and (c),(d) vertical subgrid fluxes of the moist conserved variables. The dashed lines represent the LES and the solid lines represent the EDMF results. Shaded areas indicate the interquartile range from a 10-member EDMF ensemble. The LES fluxes include only the resolved part. All the results are centered on the second hour (red lines), fourth hour (green lines), and seventh hour (blue lines). (e),(f) Contribution from the eddy diffusivity (green), updraft mass flux (blue), downdraft mass flux (yellow), mass flux from subsiding environment (magenta), and the sum of all components (red) for hour 7. 

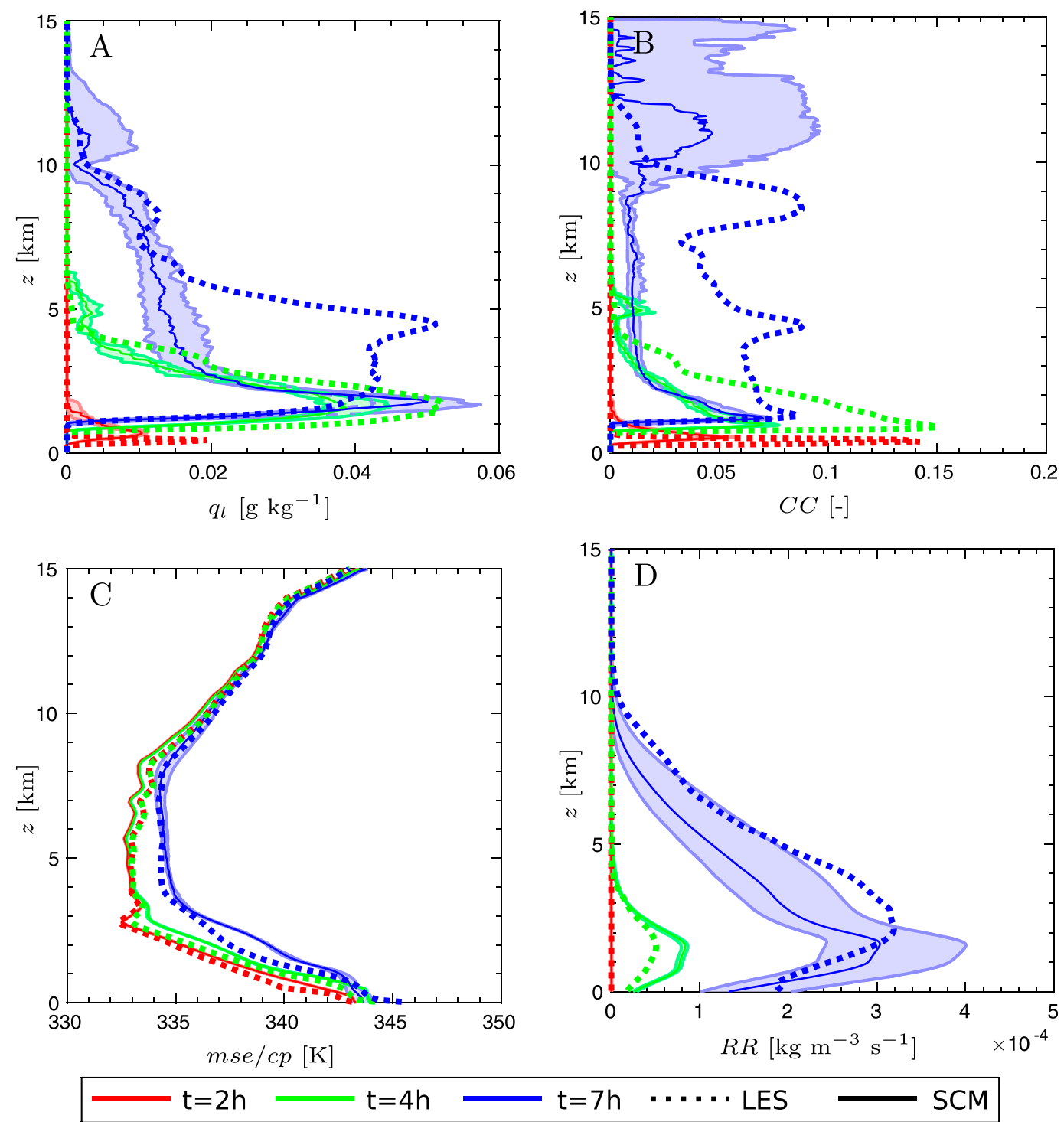

FIG. 14. LBA case. Profiles of hourly averaged (a) cloud water mixing ratio (sum of liquid and ice), (b) cloud cover, (c) moist static energy, and (d) precipitation rate. The dashed lines represent LES and the solid lines represent the EDMF results. Shaded areas indicate interquartile range from 10-member EDMF ensemble. All the results are hourly averaged values around the second hour (red), fourth hour (green), and seventh hour (blue).

The evolution of MSE is similar to that for the LBAnp case, with the main disparities present in the subcloud layer: the EDMF model tends to provide too much mixing, especially for the moisture field. On the other hand, EDMF reproduces the profile of precipitation rate quite accurately.

To understand the invigoration of convection by cold pools, Fig. 15 shows a comparison of the cloudtop heights and the precipitation rates for the LBA and LBAnocp experiments. As already discussed by Kurowski et al. (2018), the LES cold pools start to invigorate convection after the fourth hour (which is approximately when cold pools start to invigorate convection in the EDMF too; see Fig. 12). The LBAnocp case is associated with a much shallower convective layer, which never exceeds the height of $8 \mathrm{~km}$, resulting in much weaker surface precipitation. As shown in Fig. 15, the difference between the LES LBA and LBAnocp experiments for surface precipitation and cloud-top height is well reproduced by the EDMF model. Therefore, a simple EDMF parameterization of the cold pool effects still allows for a physically sound representation of the transition from shallow to deep convection. We performed two 

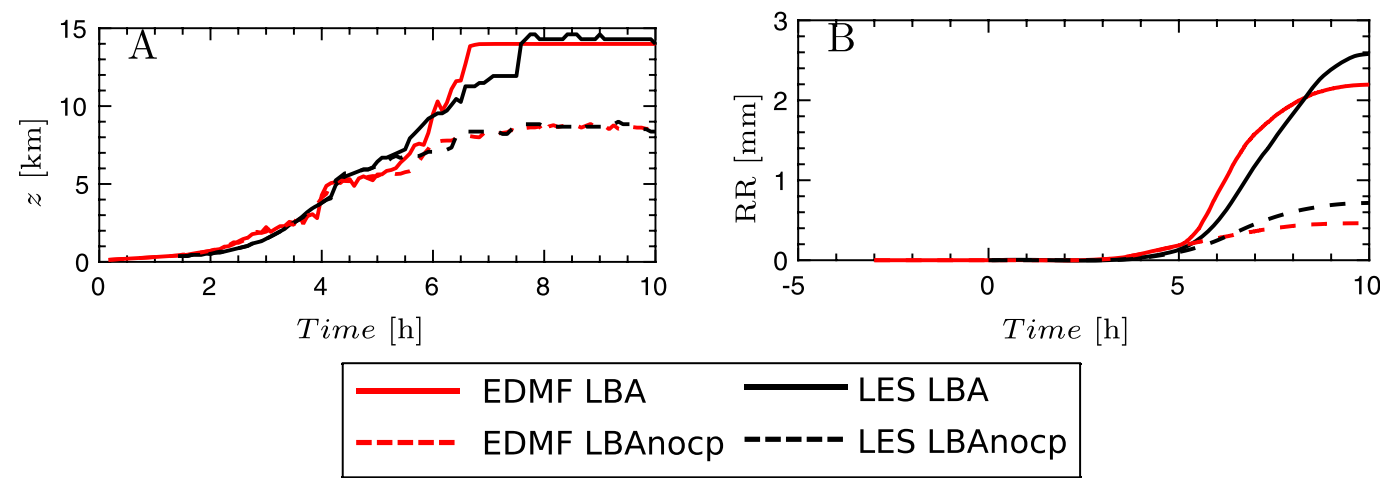

FIG. 15. The LBA and LBAnocp cases. Time series of the (a) cloud-top height and (b) cumulative surface precipitation. Black lines represent the LES result and red lines denote the ensemble mean from 10 EDMF simulations. Solid lines represent the LBA results and dashed lines represent the LBAnocp results.

complementary experiments to investigate how cold pools invigorate convection in EDMF (not shown). In the first experiment, cold pool activity affects only the entrainment rate for the updrafts and downdrafts, as governed by Eq. (30), while the surface updraft conditions remain unaffected by cold pool activity. In the second experiment, only the surface updraft conditions are affected by cold pools [as governed by Eq. (31)] while the entrainment rate is not affected. The results indicate that the primary role of cold pools is in decreasing the entrainment rate, while the modification of surface updraft conditions is less significant.

\section{Summary and conclusions}

A unified parameterization of the boundary layer, shallow convection, and deep convection, suitable for low-resolution weather and climate models, has been developed. The new parameterization is based on the stochastic multiplume eddy-diffusivity/massflux (EDMF) model (Suselj et al. 2013, 2019), which in this work has been extended to also represent precipitating convection. In the EDMF model, the subgrid domain is decomposed into a quasi-quiescent environment and a family of convective plumes, which consists of updrafts and downdrafts. The updrafts are initiated at the surface and extend up to the level of vanishing vertical velocity. Depending on their properties and individual stochastic interactions with the environment they can remain dry, or condense and eventually precipitate. Precipitating updrafts form complementary evaporatively driven downdrafts that can induce cold pool effects and modify the properties of subsequently developing plumes. These effects are currently represented by reducing the updraft entrainment rate and by enhancing the updraft surface moisture, buoyancy, and vertical velocity. The decreased entrainment rate represents the effects of convection organization, as the updrafts become less exposed to the environment, and the modification of the near-surface plume conditions represents the development of moist and buoyant near-surface patches associated with the convergence regions over which convection develops (e.g., Kurowski et al. 2018). Microphysical processes associated with the updraft dynamics are modeled with a relatively simple parameterization accounting for the liquid, mixed, and ice phases.

Each of the plumes can represent a different type of convection that can all coexist within the subgrid domain. Moreover, the new scheme facilitates seamless transitions between the dominant types of convective regimes without using any closure assumptions or arbitrary trigger functions. Instead, the transitions are obtained through the coupling between the evolving updrafts and the environment. This adaptive behavior distinguishes the proposed scheme from traditional parameterizations, which usually have to determine the regions of convective activity and a dominant type of convective regime.

The new parameterization has been implemented in a single-column model (SCM) framework, which has been used to simulate two benchmark test cases. The first case involves a slowly deepening, precipitating marine cumulus field. The second case regards the diurnal cycle of continental deep convection, which is particularly challenging as it requires a realistic representation of the transitions from dry to moist shallow and finally to deep precipitating convection. To evaluate the performance of the new parameterization, we compare the EDMF results against the reference largeeddy simulations (LESs). The results obtained are encouraging, as the new model is capable of representing the main features of convection, such as the depth of 
the convective layer and its thermodynamic and dynamical properties, as well as its evolution. The simulated surface precipitation rates are generally close to the LES results, although the onset of convection is somewhat premature. This small bias is apparent for the continental convection case, indicating that EDMF initiates convection too efficiently. To study the effects of rain formation in EDMF, we perform additional simulations without rain formation to contrast the results with the complete setup. Although the comparison reveals that EDMF is somewhat too efficient in terms of the vertical transport, the results remain in a good agreement with LES.

We are implementing the new parameterization in a GCM to verify its performance for a much broader range of atmospheric conditions. This includes investigations of the effects of the new parameterization on global circulation patterns, spatial and temporal distributions of precipitation, the diurnal cycle of convection, and the representation of longer time-scale variability in the tropics (such as the Madden-Julian oscillation). While these tests help to evaluate the parameterization, we continue to improve the parameterization within the SCM framework. In particular, it is important to examine the sensitivity of model results to key parameters (such as those defining the velocity scale of cold pools and microphysics). Other potential improvements can be obtained by (i) coupling more advanced microphysical parameterizations to the convective plumes, (ii) modeling stratiform microphysics together with a more advanced PDF-based cloud formation scheme, and (iii) introducing interactions between the convective updrafts to further represent the effects of convection organization.

Acknowledgments. The research was carried out at the Jet Propulsion Laboratory, California Institute of Technology, under a contract with the National Aeronautics and Space Administration. Parts of this research were supported by the U.S. Department of Energy, Office of Biological and Environmental Research, Earth System Modeling; the NASA MAP Program; the Office of Naval Research, Marine Meteorology Program; and the NOAA/ CPO MAPP Program. We thank three anonymous reviewers for constructive comments.

\section{APPENDIX A}

\section{Numerical Integration of the SCM Model Equations}

Time integration in the SCM model is performed as follows. At the beginning of a time step, the updraft and downdraft properties are computed as described in sections $2 \mathrm{~d}-\mathrm{f}$. To solve the updraft equations [Eq. (11)] efficiently, we neglect the source terms due to evaporation and melting of precipitation in the updrafts (i.e., $S_{q_{t}, u_{n}}^{\mathrm{ev}}, S_{\theta_{l i}}^{\mathrm{ev}}$, and $\left.S_{\theta_{l i}, u_{n}}^{\mathrm{melt}}\right)$. These source terms are, however, considered when computing the source terms for gridmean thermodynamic variables [Eq. (5)] and the vertical profile of precipitation rate [Eq. (18)]. In addition, we neglect the impact of precipitating water loading on the updraft buoyancy (in the definition of virtual potential temperature). Without these simplifications, the updraft equations describe a complex two-point boundary layer problem as the boundary conditions for prognostic variables are defined at the surface while the boundary condition of zero precipitation rate is imposed at the termination height of the updraft. The known methods for solving these types of problems require iterative approaches (Press et al. 1992). As the height of the upper boundary condition (i.e., termination height of the updraft) is a function of thermodynamic properties of the updraft, it is not straightforward how to design a converging iterative method.

Next, the values of prognostic variables in the nonconvective environment and its fractional area are evaluated with the residual approach [Eqs. (35) and (A4)]. Condensed water and cloud cover in the environment are computed as described in section $2 \mathrm{~h}$, and the values of diagnostic variables (potential temperature, water vapor, etc.) in the environment are evaluated. The mean values of diagnostic variables and source terms are computed as the area-weighted averages of the three components [Eqs. (4) and (5)]. The final step before time integration is the computation of total subgrid vertical fluxes [Eq. (7)].

For consistency and numerical stability, the prognostic SCM equations [Eqs. (1) and (2)] are integrated with a single (i.e., without any time- or process-splitting approximation) forward-in-time semi-implicit numerical solver, from the time $t$ to the time $t+\Delta t$. In this solver, the large-scale horizontal advection and source terms are treated explicitly (i.e., they are taken at the time $t$ ), ${ }^{4}$ whereas the vertical advection term is treated implicitly (taken at time $t+\Delta t$ ) and the subgrid fluxes are treated semi-implicitly. For this, the equation for subgrid-scale fluxes [Eq. (7)] is rewritten with the help of Eqs. (4), (6), and (8), so that it does not explicitly contain the values that represent environmental properties:

$$
\overline{w^{\prime} \varphi^{\prime}}=-\alpha_{\varphi} \frac{\partial \bar{\varphi}}{\partial z}+\beta \bar{\varphi}+\gamma_{\varphi},
$$

\footnotetext{
${ }^{4}$ Only the dissipation of TKE is semi-implicit.
} 
where the terms $\alpha_{\varphi}, \beta$, and $\gamma_{\varphi}$ are the functions of the updraft and downdraft properties, mean vertical velocity, mixing length scale, and TKE:

$$
\begin{aligned}
\alpha_{\varphi}= & a_{e} l_{\varphi} \sqrt{e}, \\
\beta=- & \frac{\sum_{n=1}^{N} a_{u_{n}}\left(w_{u_{n}}-\bar{w}\right)+\sum_{m=1}^{M} a_{d_{m}}\left(w_{d_{m}}-\bar{w}\right)}{a_{e}}, \\
\gamma_{\varphi}= & \sum_{n=1}^{N} a_{u_{n}} w_{u_{n}} \varphi_{u_{n}}+\sum_{m=1}^{M} a_{d_{m}} w_{d_{m}} \varphi_{d_{m}} \\
& +\frac{1}{a_{e}}\left(\sum_{n=1}^{N} a_{u_{n}} \varphi_{u_{n}}+\sum_{m=1}^{M} a_{d_{m}} \varphi_{d_{m}}\right) \\
& \times\left(\sum_{n=1}^{N} a_{u_{n}} w_{u_{n}}+\sum_{m=1}^{M} a_{d_{m}} w_{d_{m}}-\bar{w}\right),
\end{aligned}
$$

with the environmental fraction area expressed as

$$
a_{e}=1-\left(\sum_{n=1}^{N} a_{u_{n}}+\sum_{m=1}^{M} a_{d_{m}}\right) .
$$

In the solver, the mean values in the Eq. (A1) are evaluated at $t+\Delta t$, while the terms $\alpha_{\varphi}, \beta$, and $\gamma_{\varphi}$ are taken at time $t$. The time integration is expressed with a linear system of equations whose solution requires an inversion of a tridiagonal matrix, which is obtained with a simple Gaussian elimination.

\section{APPENDIX B}

\section{Numerical Integration of the Mass-Flux Equation for the Updrafts}

Suselj et al. (2014) discussed challenges related to discretizing the updraft equation [Eq. (11)] without source terms (i.e., for $S_{\varphi, u_{n}}=0$ ). In such a case, this equation represents an exponential relaxation of the updraft property $\varphi_{u_{n}}$ toward its environmental value $\bar{\varphi}$. Suselj et al. (2014) showed that, if the updraft equation is discretized with the upwind scheme, vertical grid spacing $\Delta z$ must be finer than the inverse of the entrainment rate $\left(\Delta z<\varepsilon_{u_{n}}^{-1}\right)$, or else the numerical solution for the updraft property overshoots the asymptotic environmental value (which is absolutely nonphysical). Since lateral entrainment is modeled as a random process that is in principle unlimited, if one uses the upwind differencing scheme, then an additional constraint for the entrainment rate is needed (which would be a function of the vertical resolution of the model). A second type of numerical issue arises when the source terms in Eq. (11) are nonzero and the vertical velocity becomes small (usually around the top of moist convective updrafts). There, if the last term in Eq. (11) is not discretized carefully, it can be unreasonably high. Below we derive a numerical discretization of the updraft equation that avoids these two problems. For clarity, we omit the index $u$ referring to individual updrafts of the ensemble.

We start with the updraft equation for total-water mixing ratio $q_{t, u_{n}}$ and rewrite Eq. (11) into a generalized form:

$$
\frac{\partial q_{t, u_{n}}}{\partial z}=\sum_{k=1}^{K} A_{q_{t}, u_{n}}^{k}
$$

where $K$ is the number of processes affecting the vertical distribution of $q_{t, u_{n}}$, which are symbolized by $A$. For moist precipitating convection, $K=3$ as three processes modify the updraft total-water mixing ratio: lateral entrainment $A_{q_{t}, u_{n}}^{1}=\varepsilon_{u_{n}}\left(\bar{q}_{t, u_{n}}-q_{t}\right)$, precipitation formation $A_{q_{t}, u_{n}}^{2}=S_{q_{t}, u_{n}}^{\mathrm{ac}} / w_{u_{n}}$, and precipitation evaporation $A_{q_{t}}^{3}=S_{q_{t}, u_{n}}^{\mathrm{ev}} / w_{u_{n}}$. Because we do not compute the evaporation rate simultaneously with the integration of thermodynamic variables, we omit the $A^{3}$ term in the derivation of the numerical integration scheme. For the same reasons, when solving the equation for $\theta_{l i, u_{n}}$, we omit the source term due to melting of precipitation. The vertical gradient of $q_{t, u_{n}}$ is estimated as a sum of the gradients from the processes described by $A_{q_{t}, u_{n}}^{k}$. This is a method similar to the process splitting used by atmospheric models for the time integration of physical tendencies (Williamson 2002). Therefore, the fractional contribution from the $k$ th process is given by

$$
\frac{\partial q_{t, u_{n}}^{k}}{\partial z}=A_{q_{t}, u_{n}}^{k}
$$

with the initial condition $q_{t, u_{n}}^{k}(z)=q_{t, u_{n}}(z)$.

Knowing the solution at the height $\Delta z$, one can compute the solution at the height $z+\Delta z$ as a sum of the fractional contributions:

$$
q_{t, u_{n}}(z+\Delta z)=q_{t, u_{n}}(z)+\sum_{k=1}^{K} q_{t, u_{n}}^{k}(z+\Delta z)-q_{t, u_{n}}^{k}(z),
$$

The value of $q_{t, u_{n}}^{k}(z+\Delta z)$ is obtained by analytical integration of Eq. (B2) from $z$ to $z+\Delta z$.

To compute $q_{t, u_{n}}^{1}(z+\Delta z)$, we assume that $\bar{q}_{t}$ and $\varepsilon_{u_{n}}$ are both constant between $z$ and $z+\Delta z$, with their approximate values determined at midlevels (i.e., at $z+1 / 2 \Delta z$ ). The analytic solution with the boundary condition of $q_{t, u_{n}}^{1}(z)=q_{t, u_{n}}(z)$ is given by 


$$
q_{t, u_{n}}^{1}(z+\Delta z)=q_{t, u_{n}}(z)+\left[\bar{q}_{t}-q_{t, u_{n}}(z)\right]\left(1-e^{-\varepsilon_{u_{n}} \Delta z}\right) .
$$

The source term due to autoconversion $A_{q_{t}, u_{n}}^{2}$ [see Eq. (15)] can be rewritten as

$$
\frac{\partial q_{t, u_{n}}^{2}}{\partial z}=-\frac{q_{t, u_{n}}-q_{*, u_{n}}}{\tau_{p} w_{u_{n}}} H\left(q_{t, u_{n}}-q_{*, u_{n}}\right),
$$

where $q_{*, u_{n}} \equiv q_{s, u_{n}}+q_{0}, q_{s, u_{n}}$ is the saturated water mixing ratio for the $n$th updraft, $q_{0}$ is the threshold above which precipitation occurs, and $H$ is the Heaviside step function (which is 1 for positive arguments and 0 otherwise). Analytical integration of Eq. (B5) with constant $\tau_{p}$ and constant vertical velocity (evaluated at the height $z$ ) yields

$$
\begin{aligned}
q_{t, u_{n}}^{2}(z+\Delta z)= & q_{t, u_{n}}^{2}(z)-\left[q_{t, u_{n}}(z)-q_{*, u_{n}}\right] \\
& \times\left\{1-e^{-\Delta z /\left[w_{u_{n}}(z) \tau_{p}\right]}\right\} H\left[q_{t, u_{n}}(z)-q_{*, u_{n}}\right] .
\end{aligned}
$$

The updraft equation for ice-liquid water potential temperature is discretized in a similar manner, except that the partial solution related to the precipitation formation source term is proportional to the one for the total-water mixing ratio:

$$
\theta_{l i, u_{n}}^{2}(z+\Delta z)-\theta_{l i, u_{n}}^{2}(z)=-\frac{L}{c_{p} \pi}\left[q_{t, u_{n}}^{2}(z+\Delta z)-q_{t, u_{n}}^{2}(z)\right] .
$$

Combining all terms, the final form of the numerical solution at the $z+\Delta z$ level for the updraft properties is given by the following set of equations:

$$
\begin{aligned}
q_{t, u_{n}}(z+\Delta z)= & q_{t, u_{n}}(z)-\Delta q_{t, u_{n}}^{M}\left(1-e^{-\varepsilon_{u_{n}} \Delta z}\right) \\
& -\Delta q_{t, u_{n}}^{\circ}\left\{1-e^{-\Delta z /\left[w_{u_{n}}(z) \tau_{p}\right]}\right\} H\left(\Delta q_{t, u_{n}}^{\circ}\right),
\end{aligned}
$$

$$
\begin{aligned}
\theta_{l i, u_{n}}(z+\Delta z)= & \theta_{l i, u_{n}}(z)-\Delta \theta_{l i, u_{n}}^{M}\left(1-e^{-\varepsilon_{u_{n}} \Delta z}\right) \\
& +\frac{L}{\pi c_{p}} \Delta q_{t, u_{n}}^{*}\left\{1-e^{-\Delta z\left[w_{u_{n}}(z) \tau_{p}\right]}\right\} H\left(\Delta q_{t, u_{n}}^{\circ}\right),
\end{aligned}
$$

$u_{u_{n}}(z+\Delta z)=u_{u_{n}}(z)-\Delta u_{u_{n}}^{M}\left(1-e_{u_{n}}^{-\varepsilon_{u_{n}} \Delta z / 3}\right)$,

$v_{u_{n}}(z+\Delta z)=v_{u_{n}}(z)-\Delta v_{u_{n}}^{M}\left(1-e^{-\varepsilon_{u_{n}} \Delta z / 3}\right)$,

where $\quad \Delta \varphi_{u_{n}}^{M} \equiv \varphi_{u_{n}}(z)-\bar{\varphi}(z+1 / 2 \Delta z) \quad$ for $\quad \varphi=\left\{\theta_{l i}, q_{t}\right.$, $u, v\}$ is defined as the difference between the mean and the updraft value of the corresponding variable, and $\Delta q_{t}^{\circ} \equiv q_{t, u_{n}}(z)-q_{*}(z)$ is excess of total water over the autoconversion threshold.

The solution for the updraft vertical velocity is obtained by analytically integrating Eq. (12) from $z$ to $z+\Delta z$ for constant buoyancy $B$ (taken at the height of $z+\Delta z / 2$ ) and constant entrainment rate $\varepsilon_{u_{n}}$ :

$$
w_{u_{n}}^{2}(z+\Delta z)=\left\{\begin{array}{ll}
w_{u_{n}}^{2}(z) e^{-2 b_{w} \varepsilon_{u_{n}} \Delta z}+\frac{a_{w} B_{u_{n}}}{b \varepsilon_{u_{n}}}\left(1-e^{-2 b_{w} \varepsilon_{u_{n}} \Delta z}\right), & \text { if } \quad b \varepsilon_{u_{n}} \neq 0 \\
w_{u_{n}}^{2}(z)+2 a_{w} B_{u_{n}} \Delta z, & \text { if } \quad b \varepsilon_{u_{n}}=0
\end{array} .\right.
$$

Note that the termination height of the $n$th updraft is defined at the level where $w_{u_{n}}^{2}$ is nonpositive.

\section{APPENDIX C}

\section{Numerical Integration of the Mass-Flux Equation for the Downdrafts}

The mass-flux equations for the downdrafts are integrated downward from the height where the downdraft is initialized to the surface in a similar manner to how the updraft equations are integrated from the surface to their termination height. For clarity, in the following discussion we omit the index $d$ denoting individual downdrafts. To perform this integration, we first write the evaporative source term [from Eq. (23)] in the following form:

$$
S_{q_{t}, d_{m}}^{\mathrm{ev}}=\frac{q_{s, d_{m}}-q_{v, d_{m}}}{\tau_{E, d_{m}}} H\left(q_{s, d_{m}}-q_{v, d_{m}}\right),
$$

where we defined $\tau_{E, d_{m}}$ as

$$
\tau_{E, d_{m}}^{-1} \equiv \frac{k_{e}}{q_{s, d_{m}}} \sqrt{\mathrm{RR}_{d_{m}}}
$$


and where $q_{s, d_{m}}$ is the saturated water vapor mixing ratio in the corresponding downdraft and other symbols are the same as in Eq. (23).

For integration between level $z$ to $z-\Delta z$ the final equations for downdraft properties are

$$
\begin{aligned}
& q_{t, d_{m}}(z-\Delta z)=q_{t, d_{m}}(z)-\Delta q_{t, d_{m}}^{M}\left(1-e^{-\varepsilon_{d_{m}} \Delta z}\right) \\
& -\Delta q_{t, d_{m}}^{\circ}\left\{1-e^{-\Delta z /\left[w_{d_{m}}(z) \tau_{E, d_{m}}\right]}\right\} H\left(-\Delta q_{t, d_{m}}^{\circ}\right),
\end{aligned}
$$

$$
\begin{aligned}
& \theta_{l i}(z-\Delta z)=\theta_{l i, d_{m}}(z)-\Delta \theta_{l i, d_{m}}^{M}\left(1-e^{-\varepsilon_{d_{m}} \Delta z}\right) \\
& +\frac{L}{\pi c_{p}} \Delta q_{t, d_{m}}^{\circ}\left\{1-e^{-\Delta z /\left[w_{d_{m}}(z) \tau_{E, d_{m}}\right]}\right\} \\
& \times H\left(-\Delta q_{t, d_{m}}^{\circ}\right),
\end{aligned}
$$

$$
\begin{aligned}
& u_{d_{m}}(z-\Delta z)=u_{d_{m}}(z)-\Delta u_{d_{m}}^{M}\left(1-e^{-\varepsilon_{d_{m}} \Delta z / 3}\right) \\
& v_{d_{m}}(z-\Delta z)=v_{d_{m}}(z)-\Delta v_{d_{m}}^{M}\left(1-e^{-\varepsilon_{d_{m}} \Delta z / 3}\right)
\end{aligned}
$$

where similarly to the updrafts $\Delta \varphi_{d_{m}}^{M} \equiv \varphi_{d_{m}}(z)-\bar{\varphi}(z-$ $1 / 2 \Delta z$ ) for $\varphi=\left\{\theta_{l i}, q_{t}, u, v\right\}$ is the deviation of the downdraft property from the mean, and $\Delta q_{t, d_{m}}^{\circ} \equiv q_{t, d_{m}}$ $(z)-q_{s, d_{m}}(z)$ is the saturation excess/deficit. As the downdrafts are unsaturated, $\Delta q_{t, d_{m}}^{\circ}$ is generally negative. For numerical purposes, the additional limit that RR must be nonnegative is imposed.

The discretized equation for vertical velocity in the downdraft is

$$
w_{d_{m}}^{2}(z-\Delta z)= \begin{cases}w_{d_{m}}^{2}(z) e^{-2 \beta_{d_{m}} \Delta z}-\frac{a_{w} B_{d_{m}}}{\beta_{d_{m}}}\left(1-e^{-2 \beta_{d_{m}} \Delta z}\right), & \text { if } \beta_{d_{m}} \neq 0 \\ w_{d_{m}}^{2}(z)-2 a_{w} B_{d_{m}} \Delta z, & \text { if } \quad \beta_{d_{m}}=0\end{cases}
$$

where buoyancy $B_{d_{m}}$ is evaluated at a midlevel $z-\Delta z / 2$ and parameter $\beta_{d_{m}}$ is defined as

$$
\beta_{d_{m}}=b_{w} \varepsilon_{d_{m}}+\frac{1}{2(z-\Delta z)} \max \left[1-\exp \left(\frac{z-\Delta z}{z_{00}}-1\right), 0\right] .
$$

\section{REFERENCES}

Angevine, W. M., 2005: An integrated turbulence scheme for boundary layers with shallow cumulus applied to pollutant transport. J. Appl. Meteor., 44, 1436-1452, https://doi.org/ 10.1175/JAM2284.1.

— J. Olson, J. Kenyon, W. I. Gustafson, S. Endo, K. Suselj, and D. D. Turner, 2018: Shallow cumulus in WRF parameterizations evaluated against LASSO large-eddy simulations. Mon. Wea. Rev., 146, 4303-4322, https://doi.org/10.1175/MWR-D18-0115.1.

Arakawa, A., and W. H. Schubert, 1974: Interaction of a cumulus cloud ensemble with the large-scale environment, part I. J. Atmos. Sci., 31, 674-701, https://doi.org/10.1175/1520-0469(1974)031<0674: IOACCE $>2.0$.CO;2.

Bechtold, P., J. W. M. Cuijpers, P. Mascart, and P. Trouilhet, 1995: Modeling of trade wind cumuli with a low-order turbulence model: Toward a unified description of $\mathrm{Cu}$ and $\mathrm{Sc}$ clouds in meteorological models. J. Atmos. Sci., 52, 455-463, https:// doi.org/10.1175/1520-0469(1995)052<0455:MOTWCW>2.0.CO;2. , E. Bazile, F. Guichard, P. Mascart, and E. Richard, 2001: A mass-flux convection scheme for regional and global models. Quart. J. Roy. Meteor. Soc., 127, 869-886, https://doi.org/ 10.1002/qj.49712757309.
— M. Köhler, T. Jung, F. Doblas-Reyes, M. Leutbecher, M. J. Rodwell, F. Vitart, and G. Balsamo, 2008: Advances in simulating atmospheric variability with the ECMWF model: From synoptic to decadal time-scales. Quart. J. Roy. Meteor. Soc., 134, 1337-1351, https://doi.org/10.1002/qj.289.

Bhattacharya, R., S. Bordoni, K. Suselj, and J. Teixeira, 2018: Parameterization interactions in global aquaplanet simulations. J. Adv. Model. Earth Syst., 10, 403-420, https://doi.org/10.1002/ 2017MS000991.

Böing, S. J., H. J. J. Jonker, A. P. Siebesma, and W. Grabowski, 2012: Influence of the subcloud layer on the development of a deep convective ensemble. J. Atmos. Sci., 69, 2682-2698, https:// doi.org/10.1175/JAS-D-11-0317.1.

Bony, S., and J.-L. Dufresne, 2005: Marine boundary layer clouds at the heart of tropical cloud feedback uncertainties in climate models. Geophys. Res. Lett., 32, L20806, https://doi.org/10.1029/ 2005GL023851.

Boville, B. A., P. J. Rasch, J. J. Hack, and J. R. McCaa, 2006: Representation of clouds and precipitation processes in the Community Atmospheric Model (CAM3). J. Climate, 19, 2184-2198, https://doi.org/10.1175/JCLI3749.1.

Bretherton, C. S., and S. Park, 2009: A new moist turbulence parameterization in the Community Atmosphere Model. J. Climate, 22, 3422-3448, https://doi.org/10.1175/2008JCLI2556.1.

Cesana, G., D. E. Waliser, X. Jiang, and J. F. Li, 2015: Multimodel evaluation of cloud phase transition using satellite and reanalysis data. J. Geophys. Res. Atmos., 120, 7871-7892, https:// doi.org/10.1002/2014JD022932.

Cheinet, S., 2003: A multiple mass-flux parameterization for the surface-generated convection. Part I: Dry plumes. J. Atmos. Sci., 60, 2313-2327, https://doi.org/10.1175/1520-0469(2003) $060<2313$ :AMMPFT>2.0.CO;2.

- 2004: A multiple mass flux parameterization for the surfacegenerated convection. Part II: Cloudy cores. J. Atmos. Sci., 61, 
1093-1113, https://doi.org/10.1175/1520-0469(2004)061<1093: AMMFPF $>2.0 . \mathrm{CO} ; 2$.

_ diffusivity parameterization of cloud-topped boundary layers. Geophys. Res. Lett., 30, 1930, https://doi.org/10.1029/ 2003 GL017377.

Couvreux, F., and Coauthors, 2015: Representation of daytime moist convection over the semi-arid tropics by parametrizations used in climate and meteorological models. Quart. J. Roy. Meteor. Soc., 141, 2220-2236, https://doi.org/10.1002/ qj. 2517.

D'Andrea, F., P. Gentine, A. K. Betts, and B. R. Lintner, 2014: Triggering deep convection with a probabilistic plume model. J. Atmos. Sci., 71, 3881-3901, https://doi.org/10.1175/JAS-D13-0340.1.

Deardorff, J. W., 1980: Stratocumulus-capped mixed layers derived from a three-dimensional model. Bound.-Layer Meteor., 18, 495-527, https://doi.org/10.1007/BF00119502.

de Roode, S. R., A. P. Siebesma, H. J. J. Jonker, and Y. de Voogd, 2012: Parameterization of the vertical velocity equation for shallow cumulus clouds. Mon. Wea. Rev., 140, 2424-2436, https://doi.org/10.1175/MWR-D-11-00277.1.

ECMWF, 2017: IFS documentation-Cy43r3: Operational implementation 11 July 2017. Part IV: Physical processes. ECMWF Rep., 189 pp.

Emanuel, K., 1991: A scheme for representing cumulus convection in large-scale models. J. Atmos. Sci., 48, 2313-2335, https://doi.org/ 10.1175/1520-0469(1991)048<2313:ASFRCC >2.0.CO;2.

Gerard, L., 2007: An integrated package for subgrid convection, clouds and precipitation compatible with meso-gamma scales. Quart. J. Roy. Meteor. Soc., 133, 711-730, https://doi.org/ 10.1002/qj.58.

Golaz, J.-C., V. E. Larson, and W. R. Cotton, 2002a: A PDF-based model for boundary layer clouds. Part I: Method and model description. J. Atmos. Sci., 59, 3540-3551, https://doi.org/ 10.1175/1520-0469(2002)059<3540:APBMFB >2.0.CO;2.

,-- , and,$- 2002 \mathrm{~b}$ : A PDF-based model for boundary layer clouds. Part II: Model results. J. Atmos. Sci., 59, 3552-3571, https://doi.org/10.1175/1520-0469(2002)059<3552:APBMFB > 2.0.CO;2.

Grabowski, W. W., 1998: Toward cloud resolving modeling of large-scale tropical circulations: A simple cloud microphysics parameterization. J. Atmos. Sci, 55, 3283-3298, https://doi.org/ 10.1175/1520-0469(1998)055<3283:TCRMOL > 2.0.CO;2.

_ - and Coauthors, 2006: Daytime convective development over land: A model intercomparison based on LBA observations. Quart. J. Roy. Meteor. Soc., 132, 317-344, https://doi.org/ 10.1256/qj.04.147.

Gregory, D., and P. R. Rowntree, 1990: A mass flux convection scheme with representation of cloud ensemble characteristics and stability-dependent closure. Mon. Wea. Rev., 118, 1483-1506, https://doi.org/10.1175/1520-0493(1990)118<1483:AMFCSW > 2.0.CO;2.

Guichard, F., and Coauthors, 2004: Modelling the diurnal cycle of deep precipitating convection over land with cloud-resolving models and single-column models. Quart. J. Roy. Meteor. Soc., 130, 3139-3172, https://doi.org/10.1256/qj.03.145.

Hohenegger, C., and C. S. Bretherton, 2011: Simulating deep convection with a shallow convection scheme. Atmos. Chem. Phys., 11, 10389-10 406, https://doi.org/10.5194/acp-11-103892011.

Hourdin, F., F. Couvreux, and L. Menut, 2002: Parameterization of the dry convective boundary layer based on a mass flux representation of thermals. J. Atmos. Sci., 59, 1105-1123, https://doi.org/10.1175/ 1520-0469(2002)059<1105:POTDCB > 2.0.CO;2.

Jakob, C., and A. P. Siebesma, 2003: A new subcloud model for mass-flux convection schemes: Influence on triggering, updraft properties, and model climate. Mon. Wea. Rev., 131, 2765-2778, https://doi.org/10.1175/1520-0493(2003)131<2765:ANSMFM> 2.0.CO;2.

Jeevanjee, N., and D. M. Romps, 2013: Convective self-aggregation, cold pools, and domain size. Geophys. Res. Lett., 40, 994-998, https://doi.org/10.1002/grl.50204.

Karlsson, J., G. Svensson, and H. Rodhe, 2008: Cloud radiative forcing of subtropical low level clouds in global models. Climate Dyn., 30, 779-788, https://doi.org/10.1007/s00382-007-0322-1.

Kessler, E., 1969: On the Distribution and Continuity of Water Substance in Atmospheric Circulations. Meteor. Monogr., No. 32, Amer. Meteor. Soc., 84 pp.

Khairoutdinov, M., and D. Randall, 2002: Similarity of deep continental cumulus convection as revealed by a three-dimensional cloud-resolving model. J. Atmos. Sci., 59, 2550-2566, https:// doi.org/10.1175/1520-0469(2002)059<2550:SODCCC $>2.0 . C O ; 2$. , and - 2006: High-resolution simulation of shallow-to-deep convection transition over land. J. Atmos. Sci., 63, 3421-3436, https://doi.org/10.1175/JAS3810.1.

Kuang, Z., and C. S. Bretherton, 2006: A mass-flux scheme view of a high-resolution simulation of a transition from shallow to deep cumulus convection. J. Atmos. Sci., 63, 1895-1909, https://doi.org/10.1175/JAS3723.1.

Kurowski, M. J., K. Suselj, W. W. Grabowski, and J. Teixeira, 2018: Shallow-to-deep transition of continental moist convection: Cold pools, surface fluxes, and mesoscale organization. J. Atmos. Sci., 75, 4071-4090, https://doi.org/10.1175/JAS-D-18-0031.1.

,-- , and 2019: Is shallow convection sensitive to environmental heterogeneities? Geophys. Res. Lett., 46, 1785-1793, https://doi.org/10.1029/2018GL080847.

Lin, Y.-L., R. D. Farley, and H. D. Orville, 1983: Bulk parameterization of the snow field in a cloud model. J. Climate Appl. Meteor., 22, 1065-1092, https://doi.org/10.1175/1520-0450(1983) 022<1065:BPOTSF $>2.0 . \mathrm{CO} ; 2$.

Lock, A. P., A. R. Brown, M. R. Bush, G. M. Martin, and R. N. B. Smith, 2000: A new boundary layer mixing scheme. Part I: Scheme description and single-column model tests. Mon. Wea. Rev., 128, 3187-3199, https://doi.org/10.1175/1520-0493(2000) $128<3187$ :ANBLMS $>2.0$. CO; 2 .

Lohmann, U., and B. Karcher, 2002: First interactive simulations of cirrus clouds formed by homogeneous freezing in the ECHAM general circulation model. J. Geophys. Res., 107, 4105, https:// doi.org/10.1029/2001JD000767.

Mahrt, L., and J. Paumier, 1984: Heat transport in the atmospheric boundary layer. J. Atmos. Sci., 41, 3061-3075, https://doi.org/ 10.1175/1520-0469(1984)041<3061:HTITAB > 2.0.CO;2.

Mapes, B., and R. Neale, 2011: Parameterizing convective organization to escape the entrainment dilemma. J. Adv. Model. Earth Syst., 3, M06004, https://doi.org/10.1029/2011MS000042.

Mellor, G. L., 1977: The Gaussian cloud model relations. J. Atmos. Sci., 34, 356-358, https://doi.org/10.1175/1520-0469(1977) $034<0356$ :TGCMR $>2.0$. CO 2 .

— , and T. Yamada, 1974: A hierarchy of turbulence closure models for planetary boundary layers. J. Atmos. Sci., 31, 17911806, https://doi.org/10.1175/1520-0469(1974)031<1791: AHOTCM $>2.0 . \mathrm{CO} ; 2$.

, and - 1982: Development of a turbulence closure model for geophysical fluid problems. Rev. Geophys. Space Phys., 20, 851-875, https://doi.org/10.1029/RG020i004p00851. 
Morrison, H., and A. Gettelman, 2008: A new two-moment bulk stratiform cloud microphysics scheme in the Community Atmosphere Model, version 3 (CAM3). Part I: Description and numerical tests. J. Climate, 21, 3642-3659, https://doi.org/ 10.1175/2008JCLI2105.1.

Neale, R. B., and Coauthors, 2012: Description of the NCAR Community Atmosphere Model (CAM 5.0). NCAR Tech. Note NCAR/TN-486+STR, 274 pp., www.cesm.ucar.edu/ models/cesm1.0/cam/docs/description/cam5_desc.pdf.

Neggers, R. A. J., 2009: A dual mass flux framework for boundary layer convection. Part II: Clouds. J. Atmos. Sci., 66, 1489-1506, https://doi.org/10.1175/2008JAS2636.1.

_ 2015: Exploring bin-macrophysics models for moist convective transport and clouds. J. Adv. Model. Earth Syst., 7, 2079-2104, https://doi.org/10.1002/2015MS000502.

— - M. Köhler, and A. Beljaars, 2009: A dual mass flux framework for boundary layer convection. Part I: Transport. J. Atmos. Sci., 66, 1465-1487, https://doi.org/10.1175/2008JAS2635.1.

Park, S., and C. Bretherton, 2009: The University of Washington shallow convection and moist turbulence schemes and their impact on climate simulations with the Community Atmosphere Model. J. Climate, 22, 3449-3469, https://doi.org/ 10.1175/2008JCLI2557.1.

Pergaud, J., V. Masson, S. Malardel, and F. Couvreux, 2009: A parameterization of dry thermals and shallow cumuli for mesoscale numerical weather prediction. Bound.-Layer Meteor., 132, 83-106, https://doi.org/10.1007/s10546-009-9388-0.

Press, W. H., B. P. Flannery, S. A. Teukolsky, and W. T. Vetterling, 1992: Numerical Recipes in Fortran 77: The Art of Scientific Computing. 2nd ed. Cambridge University Press, 933 pp.

Rangno, A. L., and P. V. Hobbs, 2005: Microstructures and precipitation development in cumulus and small cumulonimbus clouds over the warm pool of the tropical Pacific Ocean. Quart. J. Roy. Meteor. Soc., 131, 639-673, https://doi.org/ 10.1256/qj.04.13.

Rauber, R. M., and Coauthors, 2007: Rain in shallow cumulus over the ocean: The RICO campaign. Bull. Amer. Meteor. Soc., 88, 1912-1928, https://doi.org/10.1175/BAMS-88-12-1912.

Rienecker, M. M., and Coauthors, 2008: The GEOS-5 Data Assimilation System-Documentation of versions 5.0.1, 5.1.0 and 5.2.0. NASA Tech. Memo. NASA/TM-2008-104606, Vol. 27, 97 pp., http://gmao.gsfc.nasa.gov/pubs/docs/Rienecker369.pdf.

Rio, C., and F. Hourdin, 2008: A thermal plume model for the convective boundary layer: Representation of cumulus clouds. J. Atmos. Sci., 65, 407-425, https://doi.org/10.1175/ 2007JAS2256.1.

Romps, D. M., 2012: On the equivalence of two schemes for convective momentum transport. J. Atmos. Sci., 69, 3491-3500, https://doi.org/10.1175/JAS-D-12-068.1.

— vection. J. Atmos. Sci., 67, 1655-1666, https://doi.org/10.1175/ 2009JAS3307.1.

Schlemmer, L., and C. Hohenegger, 2014: The formation of wider and deeper clouds as a result of cold-pool dynamics. J. Atmos. Sci., 71, 2842-2858, https://doi.org/10.1175/JAS-D-13-0170.1.

Siebesma, A. P., and J. W. M. Cuijpers, 1995: Evaluation of parametric assumptions for shallow cumulus convection. J. Atmos. Sci., 52, 650-666, https://doi.org/10.1175/1520-0469(1995) $052<0650$ :EOPAFS $>2.0$.CO; 2 .

, and J. Teixeira, 2000: An advection-diffusion scheme for the convective boundary layer: Description and 1D results. Preprints, 14th Symp. on Boundary Layers and Turbulence, Aspen, CO, Amer. Meteor. Soc., 133-136.
— P. M. M. Soares, and J. Teixeira, 2007: A combined eddydiffusivity mass-flux approach for the convective boundary layer. J. Atmos. Sc.i, 64, 1230-1248, https://doi.org/10.1175/ JAS3888.1.

Skamarock, W. C., and Coauthors, 2008: A description of the Advanced Research WRF version 3. NCAR Tech. Note NCAR/ TN-475+STR, 113 pp., https://doi.org/10.5065/D68S4MVH.

Soares, P. M. M., P. M. A. Miranda, A. P. Siebesma, and J. Teixeira, 2004: An eddy-diffusivity/mass-flux parametrization for dry and shallow cumulus convection. Quart. J. Roy. Meteor. Soc., 130, 3365-3383, https://doi.org/10.1256/qj.03.223.

Sommeria, G., and J. W. Deardorff, 1977: Subgrid-scale condensation in models of nonprecipitating clouds. J. Atmos. Sci., 34, 344-355, https://doi.org/10.1175/1520-0469(1977)034<0344: SSCIMO $>2.0 . \mathrm{CO} ; 2$.

Sukoriansky, S., B. Galperin, and I. Staroselsky, 2005: A quasinormal scale elimination model of turbulent flows with stable stratification. Phys. Fluids, 17, 085107, https://doi.org/10.1063/ 1.2009010.

$[,-$, and V. Perov, 2006: A quasi-normal scale elimination model of turbulence and its application to stably stratified flows. Nonlinear Processes Geophys., 13, 9-22, https://doi.org/ 10.5194/npg-13-9-2006.

Suselj, K., J. Teixeira, and G. Matheou, 2012: Eddy diffusivity/mass flux and shallow cumulus boundary layer: An updraft PDF multiple mass flux scheme. J. Atmos. Sci., 69, 1513-1533, https://doi.org/10.1175/JAS-D-11-090.1.

$\longrightarrow, \ldots$, and D. Chung, 2013: A unified model for moist convective boundary layers based on a stochastic eddy-diffusivity/ mass-flux parameterization. J. Atmos. Sci., 70, 1929-1953, https://doi.org/10.1175/JAS-D-12-0106.1.

_ , T. Hogan, and J. Teixeira, 2014: Implementation of a stochastic eddy-diffusivity/mass-flux parameterization into the Navy Global Environmental Model. Wea. Forecasting, 29, 1374-1390, https://doi.org/10.1175/WAF-D-14-00043.1.

— - M. Kurowski, and J. Teixeira, 2019: On the factors controlling development of shallow convection in eddy-diffusivity/massflux models. J. Atmos. Sci., 76, 433-456, https://doi.org/10.1175/ JAS-D-18-0121.1.

Tan, Z., C. M. Kaul, K. G. Pressel, Y. Cohen, T. Schneider, and J. Teixeira, 2018: An extended eddy-diffusivity mass-flux scheme for unified representation of subgrid-scale turbulence and convection. J. Adv. Model. Earth Syst., 10, 770-800, https://doi.org/10.1002/2017MS001162.

Teixeira, J., and P. Siebesma, 2000: An mass-flux/k-diffusion approach to the parameterization of the convective boundary layer: Global model results. Preprints, 14th Symp. on Boundary Layers and Turbulence, Aspen, CO, Amer. Meteor. Soc., 231-234.

_ , and S. Cheinet, 2004: A simple mixing length formulation for the eddy-diffusivity parameterization of dry convection. Bound.-Layer Meteor., 110, 435-453, https://doi.org/10.1023/ B:BOUN.0000007230.96303.0d.

_ , and Coauthors, 2008: Parameterization of the atmospheric boundary layer. A view from just above the inversion. Bull. Amer. Meteor. Soc., 89, 453-458, https://doi.org/10.1175/ BAMS-89-4-453.

, and Coauthors, 2011: Tropical and subtropical cloud transitions in weather and climate prediction models: The GCSS/WGNE Pacific Cross-Section Intercomparison (GPCI). J. Climate, 24, 5223-5256, https://doi.org/10.1175/2011JCLI3672.1.

Tiedtke, M., 1989: A comprehensive mass flux scheme for cumulus parameterization in large-scale models. Mon. Wea. Rev., 117, 
1779-1800, https://doi.org/10.1175/1520-0493(1989)117<1779: ACMFSF $>2.0 . \mathrm{CO} ; 2$.

van Zanten, M. C., and Coauthors, 2011: Controls on precipitation and cloudiness in simulations of trade-wind cumulus as observed during RICO. J. Adv. Model. Earth Syst., 3, M06001, https://doi.org/10.1029/2011MS000056.

Wagner, T. M., and H.-F. Graf, 2010: An ensemble cumulus convection parameterization with explicit cloud treatment. J. Atmos. Sci., 67, 3854-3869, https://doi.org/10.1175/2010JAS3485.1.

Williamson, D. L., 2002: Time-split versus process-split coupling of parameterizations and dynamical core. Mon. Wea. Rev., 130 2024-2041, https://doi.org/10.1175/1520-0493(2002)130<2024: TSVPSC $>2.0 . \mathrm{CO} ; 2$
Witek, M. L., J. Teixeira, and G. Matheou, 2011a: An eddy diffusivity-mass flux approach to the vertical transport of turbulent kinetic energy in convective boundary layers. J. Atmos. Sci., 68, 2385-2394, https://doi.org/10.1175/JAS-D-11-06.1.

- — — , and —, 2011b: An integrated TKE-based eddydiffusivity/mass-flux boundary layer closure for the dry convective boundary layer. J. Atmos. Sci., 68, 1526-1540, https://doi.org/10.1175/2011JAS3548.1.

Yoshimura, H., R. Mizuta, and H. Murakami, 2015: A spectral cumulus parameterization scheme interpolating between two convective updrafts with semi-Lagrangian calculation of transport by compensatory subsidence. Mon. Wea. Rev., 143, 597-621, https:// doi.org/10.1175/MWR-D-14-00068.1. 